\title{
The challenge of cerebral magnetic resonance imaging in neonates: A new method using mathematical morphology for the segmentation of structures including diffuse excessive high signal intensities.
}

\author{
Yongchao Xu ${ }^{\mathrm{c}, \mathrm{b}, \mathrm{a}}$, Baptiste Morel ${ }^{\mathrm{d}, \mathrm{b}, \mathrm{a}}$, Sonia Dahdouh ${ }^{\mathrm{b}}$, Élodie Puybareau $^{\mathrm{c}, \mathrm{e}}$, Alessio Virzi $^{\mathrm{b}}$, Hélène Urien $^{\mathrm{b}}$, \\ Thierry Géraud ${ }^{\mathrm{c}}$, Catherine Adamsbaum ${ }^{\mathrm{b}, \mathrm{f}}$, Isabelle Bloch ${ }^{\mathrm{b}}$ \\ ${ }^{a}$ The two first authors contributed equally to this work. \\ ${ }^{b}$ LTCI, Télécom ParisTech, Université Paris-Saclay, Paris, France \\ ${ }^{c}$ EPITA Research and Development Laboratory (LRDE), Le Kremlin-Bicêtre, France \\ ${ }^{d}$ Faculty of Medicine, François Rabelais University, Pediatric Radiology, CHRU Tours, France \\ ${ }^{e}$ ESIEE, Université Paris-Est, LIGM, France \\ ${ }^{f}$ Faculty of Medicine, Paris Sud University, Pediatric Radiology Department, Bicêtre Hospital APHP, Paris, France
}

\begin{abstract}
Preterm birth is a multifactorial condition associated with increased morbidity and mortality. Diffuse excessive high signal intensity (DEHSI) has been recently described on T2-weighted MR sequences in this population and thought to be associated with neuropathologies. To date, no robust and reproducible method to assess the presence of white matter hyperintensities has been developed, perhaps explaining the current controversy over their prognostic value. The aim of this paper is to propose a new semi-automated framework to detect DEHSI on neonatal brain MR images having a particular pattern due to the physiological lack of complete myelination of the white matter. A novel method for semi- automatic segmentation of neonatal brain structures and DEHSI, based on mathematical morphology and on max-tree representations of the images is thus described. It is a mandatory first step to identify and clinically assess homogeneous cohorts of neonates for DEHSI and/or volume of any other segmented structures. Implemented in a user-friendly interface, the method makes it straightforward to select relevant markers of structures to be segmented, and if needed, apply eventually manual corrections. This method responds to the increasing need for providing medical experts with semi-automatic tools for image analysis, and overcomes the limitations of visual analysis alone, prone to subjectivity and variability. Experimental results demonstrate that the method is accurate, with excellent reproducibility and with very few manual corrections needed. Although the method was intended initially for images acquired at $1.5 \mathrm{~T}$, which corresponds to usual clinical practice, preliminary results on images acquired at 3T suggest that the proposed approach can be generalized.
\end{abstract}

Keywords: Neonatal brain MRI, preterm brain MRI, semi-automatic tissue segmentation, white matter hyperintensities, mathematical morphology, max-tree representation.

Email addresses: yongchao.xu@lrde.epita.fr (Yongchao $\mathrm{Xu}$ ), baptiste.morel@univ-tours.fr (Baptiste Morel), sonia.dahdouh@gmail. com (Sonia Dahdouh), elodie.puybareau@lrde.epita.fr (Élodie Puybareau), alessio.virzi@telecom-paristech.fr (Alessio Virzì), helene.urien@telecom-paristech.fr (Hélène Urien), thierry.geraud@lrde.epita.fr (Thierry Géraud), catherine.adamsbaum@aphp.fr (Catherine Adamsbaum), isabelle.bloch@telecom-paristech.fr (Isabelle Bloch) 


\section{Introduction}

Thanks to progress in neonatology, more than $85 \%$ of premature newborns survive [50]. However, premature birth remains a leading cause of morbidity and mortality. The brain develops rapidly during the third trimester of pregnancy and can be explored precisely by magnetic resonance imaging (MRI) in the prenatal and the postnatal periods [38, 49, 53]. Indeed, automated quantification of the cortical folding in a population of preterms, newborns and infants has been investigated in [16], showing promising results. This highlights the need for robust knowledge of normal versus pathological patterns in terms of volume, morphology, and signal intensities. An important issue related to premature newborns is the presence of excessive white matter hyperintensities (WMH) on T2weighted sequences, known also as diffuse excessive high signal intensities (so called DEHSI), initially described in [12] then in [60], and recently correlated with neuropathlogy in [40]. Yet, the prognostic value of DEHSI continues to be debated and is very controversial, ranging from a normal transient feature to a predictive factor for a mental retardation, e.g. [6, 24, 39]. A hypothesis to explain this controversy may be the lack of robustness and reproducibility in the assessment itself of the presence of DEHSI [33]. A reproducible automatic (or semiautomatic) segmentation of DEHSI may benefit from the segmentation of newborn brain MR images into different brain tissues, i.e., cortical gray matter (CoGM), basal ganglia and thalami (BGT), white matter (WM), ventricles (Vent), and cerebrospinal fluid (CSF). Furthermore, the segmentation of newborn brain MR images also provides new, quantitative information [11, 46] about the maturation of the brain: in particular the gyration process [17] and the myelination process [13, 55].

Counsell et al. [13] have reported that myelination was evident in numerous gray and white matter structures in the very preterm brain. For example, myelin was observed for example in cerebellar vermis and peduncles, dentate nucleus, ventrolateral nuclei of the thalamus at 28 weeks gestational age. Myelin was then visualized in the corona radiata, posterior limb of the internal capsule, corticospinal tracts of the precentral and postcentral gyri, and lateral geniculate bodies at 36 weeks gestational age. So, the white matter is physiologically mainly unmyelinated at term equivalent age and thereby associated with

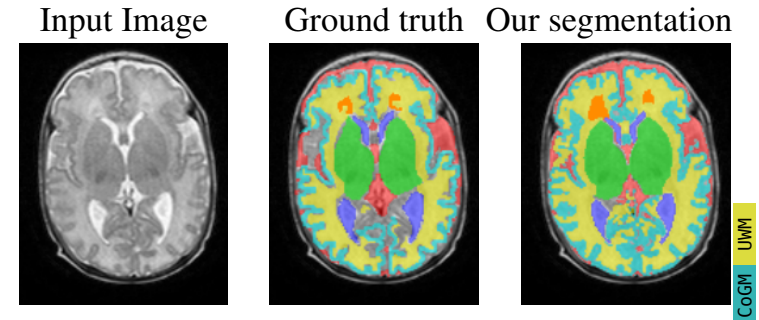

(a) On an axial T2-weighted image acquired with 1.5T MRI scanner.
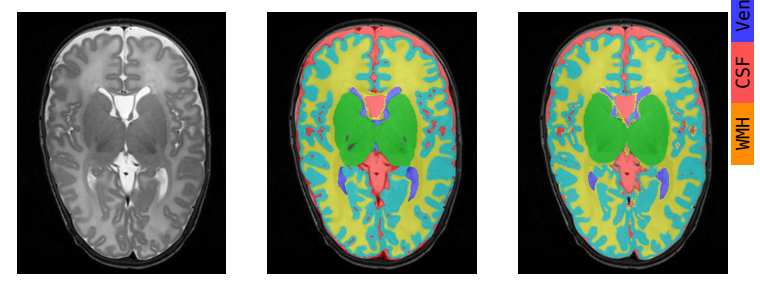

(b) On an axial T2-weighted image acquired with 3T MRI scanner.

Figure 1. Some examples of segmentation results. Note that not all the pixels inside the intracranial cavity (ICC) are manually segmented for the $1.5 \mathrm{~T}$ image in (a).

a particular signal intensity. As mentioned in some of the cited references, the contrast between gray and white matter seems to be inverted compared to children after 2 years of age and adults. The lack of myelination in neonates also has major implications to determine the presence of white matter hyperintensities because of low image contrast between normal and abnormal appearing white matter.

Therefore a reproducible and accurate method for segmenting MR images of neonatal brains on daily acquisition examinations at 1.5 Teslas $(\mathrm{T})$ would be a powerful tool for improving medical interpretation and reproducibility.

Of major concern is that the white matter is physiologically partially unmyelinated in fetuses and neonates (i.e., children under 28 days of age), and thereby associated with a particular signal intensity. This is very different from what is observed in older children and adults. Indeed, neonatal brain segmentation is challenging not only because of its small size that is responsible for significant partial volume effect, but also because of its particular contrast between white and gray mat- 
ter [20, 30, 42, 58, 64]. The lack of complete myelination in neonates also has major implications to determine the presence of DEHSI because of low image contrast between normal and abnormal appearing white matter (see Figure (1). Thereby, despite the huge amount of work on segmentation (see [14] and the references therein), the methods proposed in the adult context cannot be directly used for segmenting neonatal brain images.

The difficulties related to the neonatal period impact both computer-aided segmentation and tedious manual methods. The "gold standard" for neonatal brain segmentation [42] is manual delineation, which must follow precise guidelines (e.g., the protocol proposed in [18]) to be reproducible, and which relies on anatomical landmarks when possible. Yet, due to the challenging issues mentioned above, one may have different manual segmentation results at different times, and disagreement among experts can be significant [33]. This non-reproducible issue is especially important in the evaluation of DEHSI.

Most existing semi-automatic or automatic methods for classical neonatal brain tissue segmentation are based on atlases and/or classification. For instance, atlases were developed and used in [8, 18, 47, 57, 58], in various forms (probabilistic atlases, different atlases according to age, etc.). Although some atlases are now available for children, it is difficult to have atlases for newborns, at different ages, in a period where the brain evolves rapidly [27]. More importantly, atlas-based methods are usually efficient in normal cases, but less when additional structures are present in the images (and not in the atlas). This is typically the case for white matter hyperintensities, where the above mentioned difficulty in case of changes in geometry is increased by changes in topology. Examples of classification and learning-based approaches can be found in [2, 31, 54], sometimes combined with atlases, or more recently in [32] using convolutional neural networks. Methods relying on mathematical morphology (mainly watershed and filter by reconstruction) have been proposed in [4, 20]. Some methods focus on specific tissues, such as white matter in [23], in order to detect abnormalities based on curvature analysis. More references can be found in [15, 32].

Most of the existing methods focus on images of good quality, acquired with 3T or stronger field MRI scanners. Yet, until recently, most installed MRI scanners operate at field strengths around $1.5 \mathrm{~T}$ and result in images of inferior quality (see Figure 11). The segmentation of brain MRI at $1.5 \mathrm{~T}$ is more challenging than at $3 \mathrm{~T}$ due to the lower spatial resolution, thus inducing more partial volume effect, and lower contrast. This occurs particularly in premature newborns, which have anatomical brain structures of small size. One way to obtain a higher image quality at $1.5 \mathrm{~T}$ would be to increase the acquisition duration, but it is most of the time impossible in clinical practice, including for ethical reasons. White matter abnormalities are also more difficult to identify at $1.5 \mathrm{~T}$ than at $3 \mathrm{~T}$. Besides, to the best of our knowledge, no semi-automatic nor automatic method for detecting and segmenting DEHSI is available for newborn brain images, though a lot of methods have been developed for adult brain images (see the recent MICCAI challenge on WMH segmentation ${ }^{1}$ for $3 \mathrm{~T}$ MR images of adults, and [1, 3, 7, 19, 25, 26, 44, 59] and the references therein).

In this paper, we propose a complete pipeline dedicated to $1.5 \mathrm{~T}$ images for segmenting different neonatal brain tissues and white matter hyperintensities usually observed in supratentoria ${ }^{2}$ slices, extending our preliminary work for some tissues in [34]. In particular, we exclude brainstem and cerebellum from the segmentation to focus mainly on the supratentorial white matter. The proposed segmentation method sequentially extracts each brain tissue from the axial T2-weighted neonatal brain images: first cerebrospinal fluid (CSF) in the extracerebral space, then ventricles (Vent), basal ganglia and thalami (BGT), cortical gray matter (CoGM), unmyelinated white matter (UWM), and finally areas of white matter hyperintensities (i.e. DEHSI) (WMH). We rely on some morphological methods, notably the max-tree representation proposed in [43], which is a hierarchical representation of the image based on threshold decomposition. Two examples of segmentation results are depicted in Figure 1.

In order to improve the generalizability and the adaptability of the segmentation method, we propose to make it semi-automatic, which enables the user to place some markers and carry out some ordinary manual corrections. The manual interaction allows the user to better under-

\footnotetext{
http://wmh.isi.uu.nl/

"Note that "supratentorial" refers to the cerebral hemispheres, i.e., the region located above the tentorium cerebelli, which separates the cerebellum from the brain.
} 
stand and control the segmentation process based on each user's own objective and clinical application, which is an important aspect for clinical researchers and practitioners. The manual corrections allow the user to correct the segmentation process on the fly if needed, and to further improve the accuracy of the segmentation, according to each user's need. Indeed, as demonstrated in [33], variability among radiologists could be very significant. As demonstrated in Sections 3 and 4 , these manual interactions and corrections are easy to apply. Consequently, the proposed method constitutes a powerful tool for clinical research, and can serve as an efficient tool for preparing very precise annotations.

The main contributions of this paper include: 1) a semiautomatic method implemented as a complete pipeline, relying on the specific anatomy and image properties of neonates, that segments the neonatal brain MR images into different tissues: CSF, Vent, BGT, CoGM, UWM, and performs equally well on images obtained with MRI scanners at different field strengths; 2) a first method that extracts the diffuse excessive high sigal intensities (DEHSI) in neonatal brain MR images; 3) a user-friendly interface integrating the proposed method, without any critical parameter to tune and with the possibility given to the user to control each step and eventually perform minor corrections according to the application needs.

The rest of this paper is organized as follows. A global overview of the proposed method is given in 2 Section 3 is dedicated to detail the proposed segmentation pipeline, followed by a number of experimental results and discussion in Section 4 . We then conclude in Section 5.

In an effort to support and encourage reproducible research, the source code of the proposed method and a graphical interface with some ordinary manual corrections for different tissues will be freely available for research purposes, on demand (when the manuscript is published). All of the results presented in this paper were obtained with this graphical interface.

\section{Method overview}

The proposed method is based on common anatomical characteristics of different brain tissues and on their appearance on T2-weighted MR images. Let us first recall such prior knowledge.

\subsection{Anatomical characteristics in T2-weighted MR im-} ages of neonatal brain

As depicted in the manual segmentations shown in Figure 1, different neonatal brain tissues feature distinct characteristics on T2-weighted sequences. There is usually a dark gap corresponding to thin structures between the intracranial cavity (ICC) and the skin (fat/skull). The CSF and ventricles correspond to bright regions inside of the ICC which are relatively easily differentiated from their surroundings. The ventricles are approximately in the middle of the image. The BGT correspond to the dark and compact regions in the middle of each image slice; they are usually composed of two parts approximately symmetrically distributed on the left and right hemispheres. The CoGM consists of the dark and thin regions that are close to the ICC border, CSF, or the inter-hemispheric plane. The unmyelinated white matter corresponds to the regions that are darker than the CSF, but brighter than CoGM and BGT. The UWM regions are usually surrounded by CoGM, BGT, and ventricles. The DEHSI are in the white matter and slightly brighter than the normal surrounding white matter. The DEHSI are usually located close to the anterior and/or posterior ventricular horns, but could be located everywhere in the white matter.

This prior knowledge is common in the T2-weighted neonatal brain MR images no matter which scanners and acquisition parameters are used, and will be used to a great extent in the proposed segmentation method. More precisely, thanks to the dark gap between ICC and the skin, we start with extracting ICC by a morphological opening followed by a simple image thresholding. The CSF and ventricles are brighter than their surroundings, which makes them present in the max-tree representation [43], an intensity-based hierarchical representation of the image based on the inclusion relationship between the sets obtained by upper thresholdings. For each given marker (which can be easily obtained automatically or manually), we resort to the context-based energy estimator [63] whose minimum represents a meaningful region to be selected as the segmented tissue. This concept is also adopted to extract the BGT, which are present in a modified max-tree constructed on the image of difference between area closing and the original image. The remaining UWM (including DEHSI) and CoGM have relatively different intensities. We use Otsu's threshold [37] method on local regions to separate them. The DEHSI 


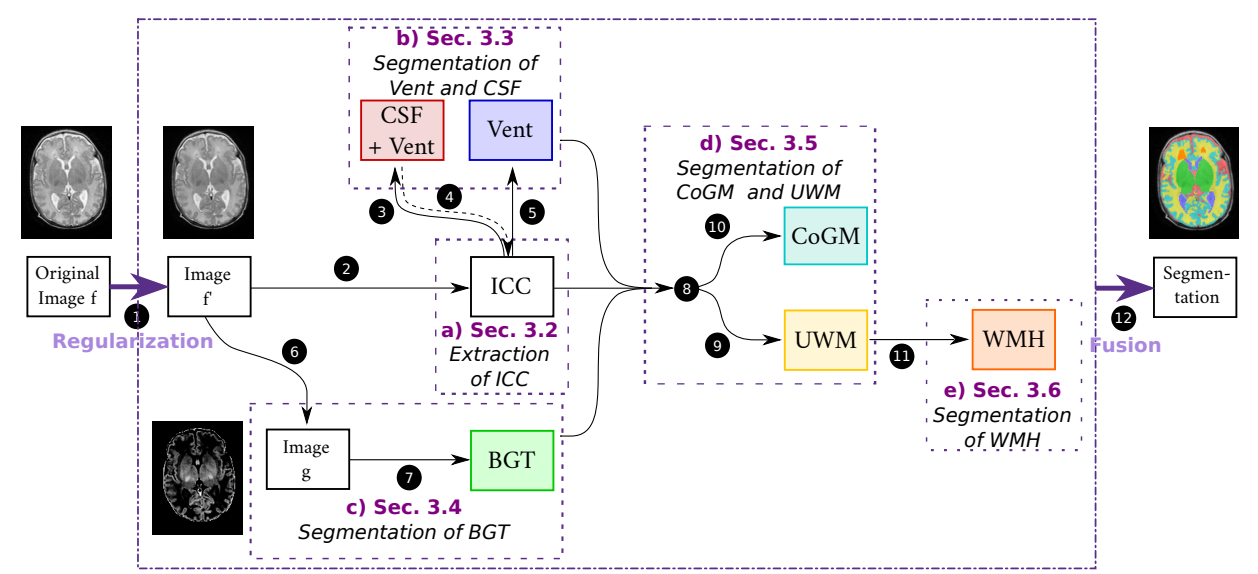

Figure 2. Overview of the proposed pipeline for the segmentation of different neonatal brain tissues. The process is performed in sequential order from Step 1 to Step 12 (Step 4 for ICC refinement being optional). The blocks (a) to (e) are detailed respectively in Sections 3.2 to 3.6

are also present in the max-tree, since they correspond to regions that are brighter than the surroundings, by definition. Consequently, we rely again on the max-tree representation and context-based energy estimator for DEHSI segmentation.

\subsection{Proposed pipeline}

An overview of the proposed pipeline is depicted in Figure 2. The T2-weighted image $f$ is first smoothed by anisotropic diffusion [41] to reduce image noise. This filtered image $f^{\prime}$ is considered as the input for the rest of the pipeline summarized as follows:

a) Extract the intracranial cavity (ICC) as the region of interest (see Section 3.2).

b) Extract the CSF and ventricles using the max-tree and the context-based energy based on the selection strategy relying on markers (see Section 3.3 .

c) Extract the BGT using a modified max-tree built on the image of difference between area closing $\phi_{a}\left(f^{\prime}\right)$ and $f^{\prime}$ (see Section 3.4).

d) Separate the gray matter and white matter from the remaining tissues based on the optimal histogrambased thresholding proposed in [37] (see Section 3.5). e) Extract the white matter hyperintensities using again the max-tree and the context-based energy (see Section 3.6.

Note that the input $\mathrm{T} 2$-weighted volumes at $1.5 \mathrm{~T}$ are strongly anisotropic and the slice thickeness is too large to allow for a true 3D processing with acceptable spatial consistency between slices. Therefore the proposed pipeline is applied in $2 \mathrm{D}$ on the set of axial slices.

\section{Details of the proposed segmentation method}

\subsection{Forewords about some morphological tools}

Some morphological operators and the max-tree representation. The most fundamental operators in mathematical morphology are the dilation $\delta_{B}$ and the erosion $\varepsilon_{B}$ [45], relying on a structuring element $B$. These operators are adjoint and dual. Two other dual fundamental operators are the opening $\gamma_{B}=\delta_{B} \circ \varepsilon_{B}$ and the closing $\phi_{B}=\varepsilon_{B} \circ \delta_{B}$ [45]. The effects of these fundamental morphological operators depend on the shape and size of the structuring element. In mathematical morphology, the area opening $\gamma_{a}$ and its dual operator, the area closing $\phi_{a}$ [51, 52], do not rely on any structuring element. They belong to the more general morphological operators named connected operators [43]. An area opening (respectively closing) consists in filtering out small bright (respectively dark) regions from the image. 


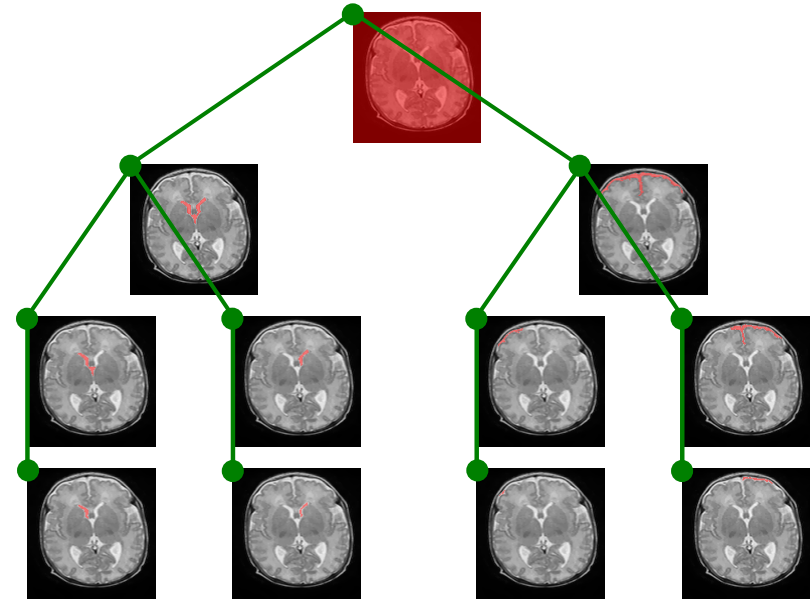

Figure 3. Partial max-tree representation of the image given in Figure 1 a). Each node (i.e., green circle) of the tree represents a connected component (i.e., red region) in the upper level sets $\mathcal{X}_{\lambda}$; note that only a very small subset of nodes of the max-tree are shown. We can observe that, starting from the leaves (bottom) and going up to the root of the tree (top), the components grow and merge; some components correspond to anatomical objects.

A popular implementation of the connected operators relies on hierarchical representations of the image based on threshold decompositions, such as the upper level sets. For any $\lambda \in \mathbb{R}^{+}$or $\mathbb{Z}^{+}$, the upper level sets $\mathcal{X}_{\lambda}$ of an image $f: \Omega \rightarrow \mathbb{R}^{+}$or $\mathbb{Z}^{+}$is defined by $\mathcal{X}_{\lambda}(f)=\{p \in \Omega \mid f(p) \geq$ $\lambda$. Upper level sets have a natural inclusion structure: $\forall \lambda_{1}, \lambda_{2}, \lambda_{1} \leq \lambda_{2} \Rightarrow \mathcal{X}_{\lambda_{1}} \supseteq \mathcal{X}_{\lambda_{2}}$, which leads to the maxtree representations of an image, first introduced in [43]. An example of the max-tree is depicted in Figure 3 Each node (i.e., green circle) of the max-tree corresponds to a connected component (i.e., red region) in $\mathcal{X}_{\lambda}$. The parenthood is given by the inclusion relationship (i.e., green lines) between the regions in the tree. Note that only a simplified tree is shown in Figure 3

In this paper, we use the immersion algorithm [5, 36] based on the union-find process [48] to construct the maxtree. This algorithm is depicted in Figure 4 by the black part, where $\mathcal{N}(p)$ denotes the set of neighboring pixels of pixel $p$. The construction process starts with sorting the pixels in increasing order of intensity, i.e. root to leaves order (see line 9 in Figure 4(b)), then relies on the unionfind process (in reverse order) to merge disjoint sets (see Figure 4(a), initially, each pixel is considered as a singleton) to form a tree structure, followed by a canoniza-

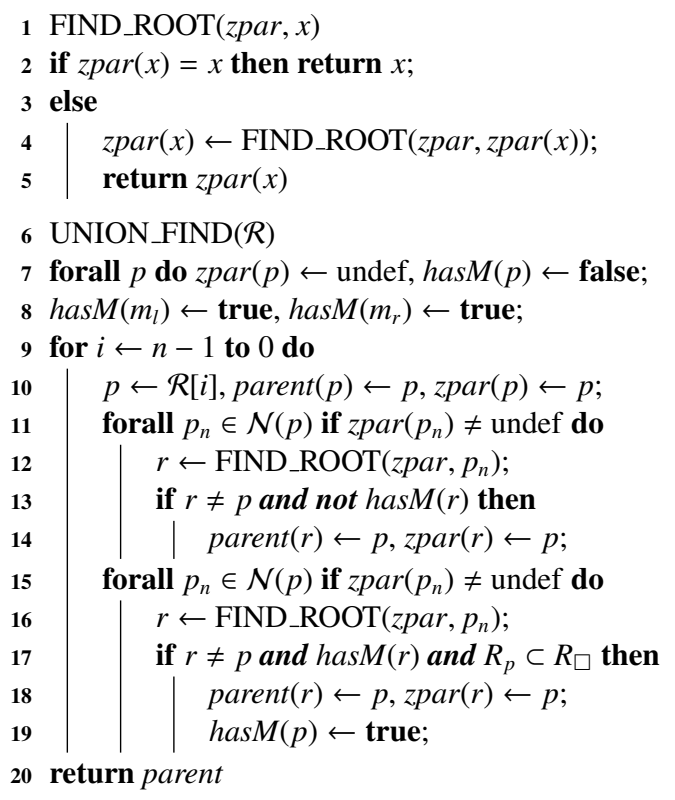

(a) Modified union-find process.

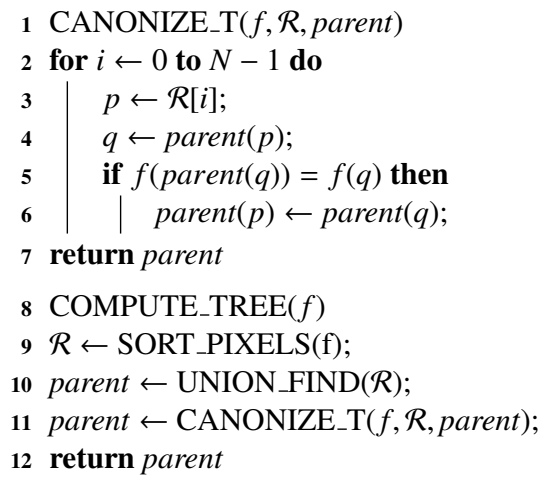

(b) Tree construction.

Figure 4. Tree construction (b) relying on a modified union-find process (a) - modifications are in red. One can see that computing such a tree-based representation of an image can be done with a very few lines of code.

tion process to compute the final tree (see line 11 in Figure $4(\mathrm{~b})$ ). The time complexity is quasi-linear with respect to the number of pixels $n$. The interested reader can refer to [10] for more details about the max-tree computation. 
Context-based energy for extracting objects from the maxtree. Following the scale-space theory [21], and since the parent of a node is a larger region, the max-tree representation is a multiscale image representation that provides a reduced search space for object spotting. As described in Section 2.1, several neonatal brain tissues are present in the max-tree representation, then segmenting them amounts to spot objects from the search space given by the max-tree.

Object spotting from a search space is usually achieved by selecting the "most likely" objects based on a measurement characterizing the objects of interest. In this paper, we use the context-based energy introduced in [63]. It is inspired by the error term of the cartoon segmentation model [35]. Given a region $R$, let us consider the error when $f$ is approximated by $\bar{f}(R)$ in region $R$, where $\bar{f}(R)$ is the mean value of $f$ in this region: $V(f, R)=$ $\sum_{p \in R}(f(p)-\bar{f}(R))^{2}$, which can be interpreted as a segmentation error. Let $\partial R$ be the boundary of region $R$. We define the interior context region $\mathcal{R}_{i n}^{\epsilon}(\partial R)$ and exterior context region $\mathcal{R}_{\text {out }}^{\epsilon}(\partial R)$ as the sets of points at a distance less than $\epsilon$ from $\partial R$, respectively inside and outside the region $R$. We use the measurement defined as:

$$
E(f, \partial R)=\frac{V\left(f, \mathcal{R}_{\text {in }}^{\epsilon}(\partial R)\right)+V\left(f, \mathcal{R}_{\text {out }}^{\epsilon}(\partial R)\right)}{V\left(f, \mathcal{R}_{\text {in }}^{\epsilon}(\partial R) \cup \mathcal{R}_{\text {out }}^{\epsilon}(\partial R)\right)} .
$$

In our experiments the parameter $\epsilon$ was set to 2 pixels. This measurement is a value between 0 and 1 . Specifically, when the interior and exterior context regions have similar intensity distributions, the sum of segmentation errors in the two regions would be close to the segmentation error for the union of these two regions. Consequently, the measurement $E$ is close to 1, meaning that the context region is composed of the same one class, and that $\partial R$ is not an object contour. For the case of interior and exterior regions having very different intensity distributions, the sum of segmentation errors for the two individual regions is much lower than that of grouping them together. Therefore, the measurement $E$ is close to 0 , meaning that the boundary $\partial R$ is an object contour, and that we have two distinct classes from either side.

\subsection{Extraction of the intracranial cavity (ICC)}

To retrieve the intracranial cavity (ICC), which is a large connected region in each slice, we can use the fact

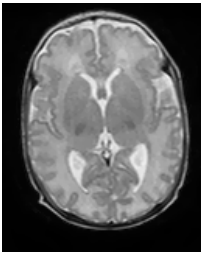

(a) $f_{1}^{\prime}$

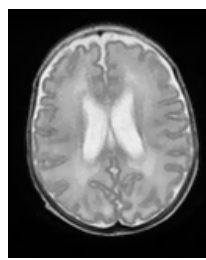

(d) $f_{2}^{\prime}$

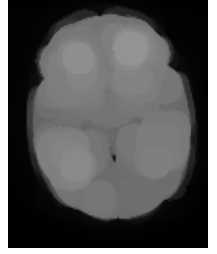

(b) $\gamma\left(f_{1}^{\prime}\right)$

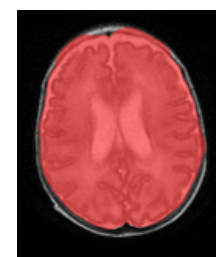

(e) $\mathrm{ICC}_{2}$

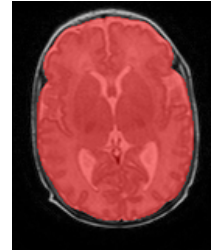

(c) $\mathrm{ICC}_{1}$

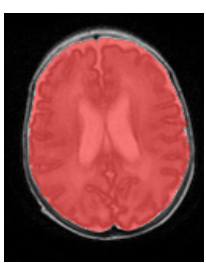

(f) $\mathrm{ICC}_{2}^{\prime}$
Figure 5. Extraction of ICC from (a) by an opening $\gamma$ (b), and a thresholding (c). On another slice (d) from the same patient, the result is (e), and an optional refinement by removing pixels outside the CSF is depicted in (f).

that the skull is a dark gap between the ICC and the extracranial tissues. We then apply a morphological opening $\gamma_{B}=\delta_{B} \circ \varepsilon_{B}$ on $f^{\prime}$ with the structuring element $B$ being a disk of $5 \mathrm{~mm}$ radius. The effect of the opening is to attenuate the bright and thin regions exterior to the ICC but connected to it (see Figures 5(a) and (d)). The resulting image is then thresholded with a thresholding value $\lambda_{I C C}$; the default value is $\lambda_{I C C}=0.3$ for an image normalized by simply dividing the intensity values by the maximal value. To clean up the binary result, we keep only the largest region, and we fill its holes. Two examples are depicted in Figures 5 (c) and (e). We apply an optional refinement step after the CSF extraction (this extraction is explained later in Section 3.3), to further remove bright and thin structures (i.e., fat/skull), that can remain strongly connected to ICC. Precisely, we define the ICC border by the set of CSF pixels having distances less than $20 \mathrm{~mm}$ to the background, and further belonging to the CSF regions whose shortest distance to the background is less than $2.5 \mathrm{~mm}$. A result of this refinement step, applied on the ICC depicted in Figure 5(e), is given in Figure 5 (f). However, note that a very precise delineation of the ICC is not a goal of the method, and that it has a minor impact on the final DEHSI extraction. 


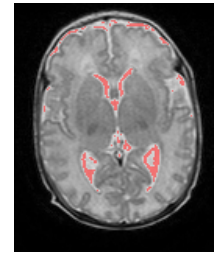

(a) $\mathbb{M}$

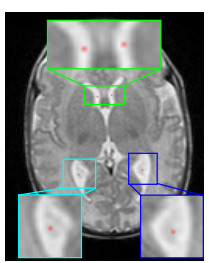

(d) $\mathbb{M}_{V_{\text {in }}}$

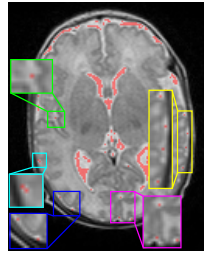

(b) Add $\mathbb{M}$

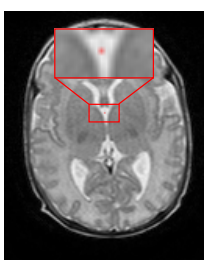

(e) $\mathbb{M}_{V_{\text {out }}}$

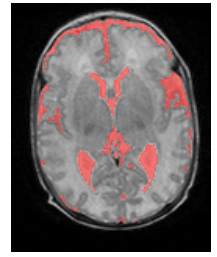

(c) $\mathrm{CSF}+\mathrm{Vent}$

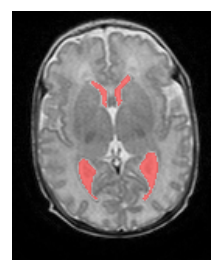

(f) LV

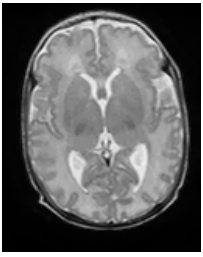

(a) $f^{\prime}$

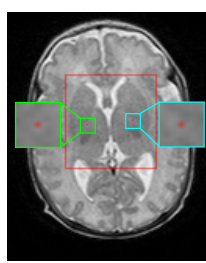

(d) $m_{l, r}, R_{\square}$

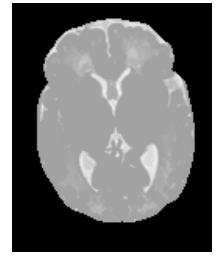

(b) $\phi_{a}\left(f^{\prime}\right)$

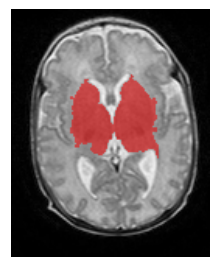

(e) BGT

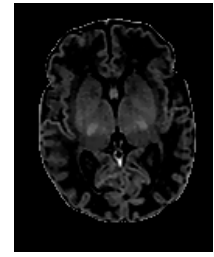

(c) $\phi_{a}\left(f^{\prime}\right)-f^{\prime}$

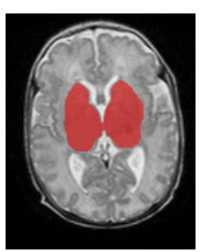

(f) $\mathrm{BGT}^{\prime}$

Figure 6. Extraction of the CSF and ventricles based on markers $\mathbb{M}$. Some CSF markers are obtained automatically by image thresholding in (a), and some are added manually in (b) to retrieve the small CSF regions. Some inside markers and outside markers for Ventricles are manually putted in (d) and (e), respectively. The extractions in (c) and (f) are achieved by object spotting on max-tree representation with their corresponding markers.

\subsection{Extraction of CSF and ventricles}

The extraction of CSF and ventricles amounts to identifying the corresponding nodes in the max-tree representation. Following the prior knowledge about CSF and ventricles being brighter than their surroundings, described in Section 2, it is easy to obtain some inside markers $\mathbb{M}$ for the CSF and ventricle regions by thresholding the image with a large value $\lambda_{m}$. Specifically, we first normalize the image into the range between 0 and 1 by dividing the pixel intensities by their maximal value. The default thresholding value for $\lambda_{m}$ is set to 0.85 . This thresholding process generates inside markers $\mathbb{M}$ (see Figure 6(a)) that lie in most of the CSF and ventricle regions. One may also manually add some markers (see Figure 6(b)) for some small bright regions if a very precise segmentation is required. For each marker $m \in \mathbb{M}$, the region that has the smallest context-based energy among all the ancestor regions of $m$ is extracted as one CSF or ventricle region (see Figure 6(c)). It is noteworthy that the CSF and the ventricle regions are not yet separated at this stage. An example is illustrated in Figure $6(\mathrm{a}-\mathrm{c})$.

In order to separate the ventricle regions and the CSF, we begin with setting inside manual markers $\mathbb{M}_{V_{i n}}$ (see

Figure 7. An example of BGT extraction based on the modified max-tree depicted in Figure 4 constructed on the difference of $f^{\prime}$ and its area closing $\phi_{a}\left(f^{\prime}\right)$ in (b). We set manually two inside markers and a bounding rectangle in (d) to limit the area of BGT segmentation. A regularization is applied to further refine such extracted BGT in (e), resulting the final BGT segmentation in (f). See the corresponding text for more details.

Figure 6(d)) and outside manual markers $\mathbb{M}_{V_{\text {out }}}$ (see Figure 6(e)) for the ventricles. This is followed by disabling the ancestor regions of $\mathbb{M}_{V_{\text {out }}}$ in the max-tree. Then the same selection process as above is repeated for the nondisabled nodes in the max-tree. An example is given in Figure 6(d-f).

\subsection{Extraction of basal ganglia and thalami (BGT)}

For the extraction of BGT, being compact and dark regions as described in Section 2.1, we rely on a novel modified max-tree built on the difference between the original image and its area closing (see Figure 7 (b)) with a large area value (set to $66 \%$ of the ICC size, based on usual proportions of these anatomical structures). It is easier to extract the BGT on this difference image than on the original one (see Figure 7(c)). Yet, the whole BGT region is not perfectly represented by a node of the max-tree. A novel modified max-tree is proposed to solve this based on two inside markers $m_{l}$ and $m_{r}$ in the left and right parts of BGT, and on a constraint rectangle $R_{\square}$ that encloses the BGT. For the experiments in this paper, the two markers and the constraint rectangle are set manually (see Figure $7(d))$. Compared to the classical max-tree, the modification lies in the union-find process by forbidding the 
merging of the region containing the inside markers with the region which is not completely inside $R_{\square}$. The algorithm is depicted in Figure 4 by adding the red part to the classical union-find process (black part). More precisely, for each current pixel $p$ during the union-find process, we merge it with its neighboring regions that do not contain the markers (see lines 11-14 in Figure 4(a)). Then, for its neighboring region containing the markers (see lines 1519 in Figure $4(\mathrm{a})$ ), if the former merged region $R_{p}$ is completely inside the constraint rectangle, we merge them. Otherwise, we process the next pixel in the propagation order. For each marker, the largest region containing it in the modified max-tree is selected as the extracted BGT region (see Figure 7(e)) .

We further apply a regularization based on maximal Cheeger set proposed in $[9]^{3}$ to smooth the contour of BGT. Basically, the idea is to approximate the region of the BGT (illustrated in Figure 7(e)) by the closest shape having a high compacity. The smoothed BGT region is displayed in Figure 7 (f).

\subsection{Extraction of gray matter and white matter}

After subtracting the CSF, the ventricles, and the BGT from the ICC, the residual image (or residue, for short) is composed of gray matter and white matter. The gray levels of these two tissues are relatively separable. A simple way to separate them is to analyze the histogram of the residue in order to estimate an optimal threshold. This can be efficiently achieved by Otsu's thresholding method [37]. Yet, due to an important overlap between the distributions of white and gray matters (see Figure 8 a)), direct thresholding on the entire ICC usually fails. We thus propose to divide the residue into small blocks (e.g., $10 \times 8$ blocks); these blocks are depicted in Figure 9(b). We then apply the Otsu's method on each small block, which improves the separability of gray matter and white matter. Indeed, as depicted in Figure 8 (b-d), the overlapping of distributions of gray levels for gray matter and white matter inside each small block is not significant. However, it may happen that a small block contains only white matter; in this case, the Otsu's method applied on this block yields some false gray matter pixels. We then

${ }^{3}$ The source code is available on https://github.com/gpeyre/ 2009-M3AN-cheeger

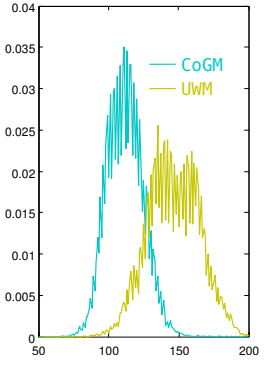

(a) On the whole image

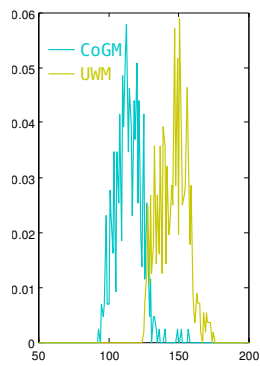

(c) On a small part 2

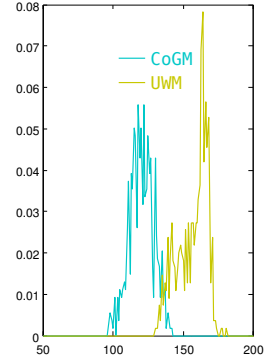

(b) On a small part 1

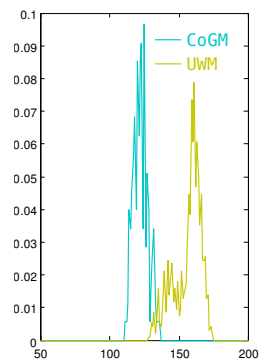

(d) On a small part 3
Figure 8. Distribution of gray levels for cortical gray matter and unmyelinated white matter.

rely on a local validation step to solve this issue. Precisely, for each previously extracted gray matter pixel $p_{g}$, we apply the Otsu's method on a local window centered at $p_{g}$. The local window is large enough (e.g., $41 \times 41$ pixels) so that it contains both gray matter and white matter; such a local window is depicted in Figure 9.c). If the second thresholding process again predicts pixel $p_{g}$ as gray matter, the pixel $p_{g}$ is confirmed as a gray matter pixel. Otherwise, the pixel $p_{g}$ is identified as a white matter pixel. A result of the cortical gray matter extraction on the image given in Figure 9 a) is depicted in Figure 9 (d).

The rest of the residue contains only white matter. As depicted in Figure 9(e), there are some thin structures in the residue that do not belong to white matter. We apply an opening with a square of $1 \times 1 \mathrm{~mm}^{2}$ as structuring element to remove these thin structures. This yields the extracted white matter. Note that this final opening can result in a few pixels that are not assigned to any anatomical structure or tissue. An example of UWM extraction is illustrated in Figure $9(\mathrm{f})$.

\subsection{Extraction of white matter hyperintensities}




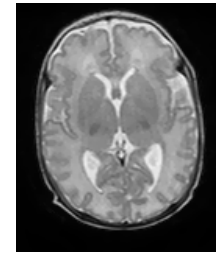

(a) $f^{\prime}$

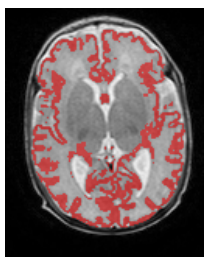

(d) CoGM

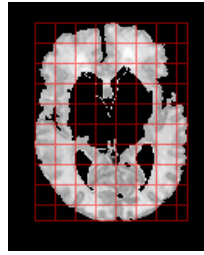

(b) Blocks

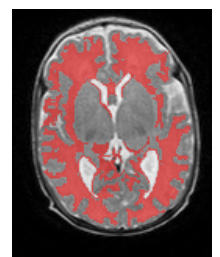

(e) Residue

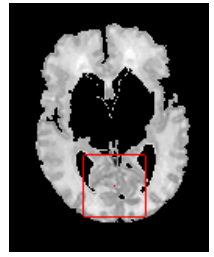

(c) Validation

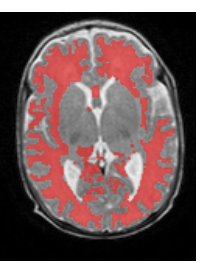

(f) UWM

Figure 9. An example of CoGM and UWM extraction. Given an image (a), a local thresholding is performed on small blocks (b), and sometimes validated on a larger area (c), to obtain a segmentation of the CoGM (d). Subtracting the CSF, the ventricles, the BGT, and the CoGM from the ICC gives the residue (e), from which the UWM is extracted (f).

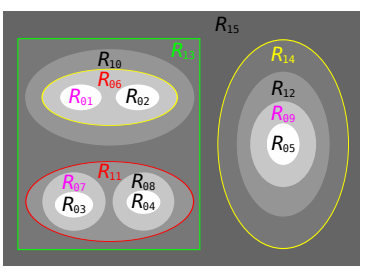

(a) Image

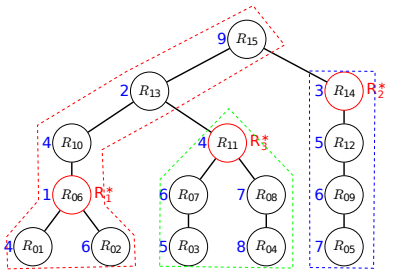

(b) Selecting and discarding

Figure 10. An example of object spotting on the max-tree based on selecting and discarding process. Spotting an object means finding the "best" tree node that corresponds to the expected object. For that, an energy is assigned to each node (depicted in blue on the left of the nodes), where minima values of the energy on the tree give candidate objects. The spotting strategy, depicted in (b), repeats the process of selecting the node $R_{i}^{*}$ having the smallest energy, and of discarding the ancestors and descendants of this node. Here, the first spotted node $R_{1}^{*}$ is $R_{06}$, which has the smallest energy; its ancestors and descendants (within the red dotted line) are discarded. The smallest energy for the remaining nodes is 3 at node $R_{14}$ so we have $R_{2}^{*}=R_{14}$, and we discard the nodes within the blue dotted line. Last, $R_{3}^{*}=R_{11}$.

The next step aims at segmenting DEHSI from the regions corresponding to the extracted white matter (this extraction is described in the previous section). There are two main steps: 1) select a set of DEHSI candidates on the max-tree; 2) filter out some candidates based on relative intensities with their surroundings.

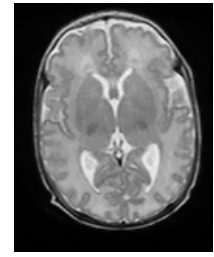

(a) Input image

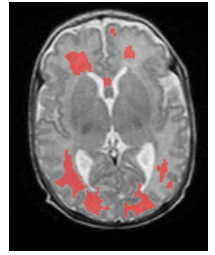

(b) Candidates

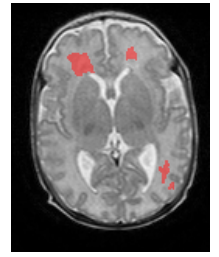

(c) Filtered Hyper
Figure 11. Extraction of hyperintensities based on selecting regions from the max-tree constructed on the previously extracted UWM regions. This is followed by a validation process (see text for details) that provides the result (c).

Let us denote by $f_{w m}^{\prime}$ the input image masked with the extracted white matter regions (0 elsewhere). According to the anatomical characteristics described in Section 2 , the DEHSI are disjoint regions, and are present in the max-tree of $f_{w m}^{\prime}$. We rely on the context-based energy to identify the DEHSI. Yet, as manual segmentation of hyperintensities is very subjective, it is consequently difficult to obtain their inside markers. We use an alternative selecting and discarding process to extract them. More precisely, we first spot the "most likely" region $R_{1}^{*}$ having the minimum context energy among all the regions in the tree, and discard all the ancestors and descendants of $R_{1}^{*}$. Then we retrieve a second "most likely" region $R_{2}^{*}$ having the minimum context energy among the remaining regions in the search space, and discard again its descendants and ancestors. This selecting and discarding process is repeated until all the regions are either spotted or discarded. In consequence, a set of regions $\left\{R_{i}^{*}, i=1 \ldots N\right\}$ will be spotted, where the number of spotted objects $N$ is decided by the algorithm. An example of this process is depicted in Figure 10 Note that we ignore the regions outside the extracted white matter when computing the energy.

Let us make clear that the context-based energy is very well adapted to the task of locating the "best" possible DEHSI boundaries. Indeed, a node of the max-tree corresponds to a region obtained by thresholding. The energy computed on the context, that is, on the part of the image around the region boundary, expresses how well this boundary separates the context into two distinct classes [63]. So, applied on the max-tree, it thus considers all the regions obtainable by any local threshold, and then points out the region (corresponding to the node with the 


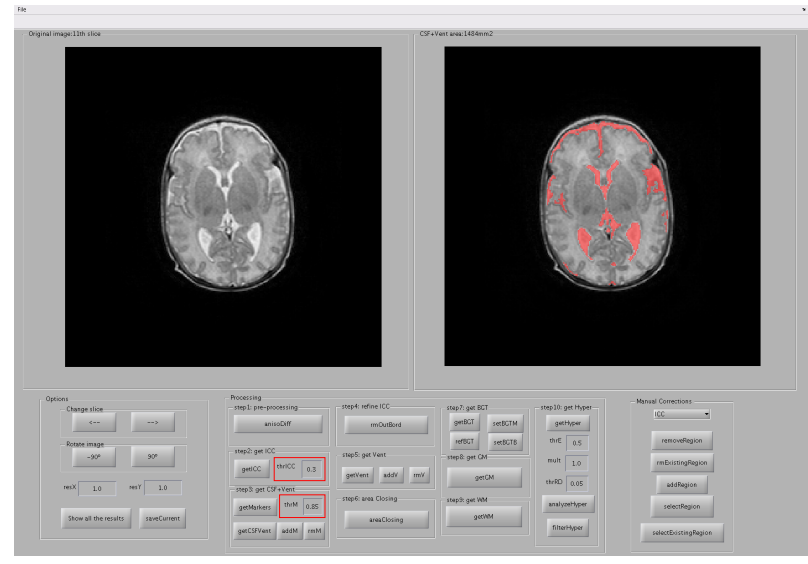

Figure 12. Illustration of the graphical user interface integrating each step of the proposed pipeline and some ordinary manual corrections.

lowest energy) whose boundary best separates locally the image into two classes. This property also applies when the boundary of a DEHSI region is very diffuse. Eventually, this spotting process yields a number of regions. Only the regions having relatively small energy values (e.g., smaller than 0.5) and high mean gray levels (e.g., larger than a given threshold $\lambda_{f}$ ) are considered as candidate DEHSI regions (see Figure 11(b)). The threshold value $\lambda_{f}$ is given by $\lambda_{f}=\overline{f^{\prime}}(U W M)+\alpha \times \sigma\left(f^{\prime}(U W M)\right)$, where $\overline{f^{\prime}}(U W M)$ denotes the mean gray level on the previously extracted UWM, $\sigma$ stands for the standard deviation, and $\alpha$ is a parameter (set to $\alpha=1$ by default).

For each candidate DEHSI region $R_{h}$, we compute the relative difference $\lambda_{h}$ of mean gray levels $\overline{f^{\prime}}$ between $R_{h}$ and its surrounding white matter regions $R_{w m}:\left(\overline{f^{\prime}}\left(R_{h}\right)-\right.$ $\left.\overline{f^{\prime}}\left(R_{w m}\right)\right) / \overline{f^{\prime}}\left(R_{w m}\right)$. A DEHSI region should have a rather important relative difference $\lambda_{h}(0.05$ by default). We use this criterion to further filter out some candidate DEHSI regions. An example is illustrated in Figure 11.

\subsection{Implementation and graphical interface with manual corrections}

The proposed pipeline has been implemented in Matlab $^{\odot}$. For the max-tree construction, as described in Section 3.1. we use the immersion algorithm [5, 36] based on union-find process depicted in Figure 4, which has a quasi-linear time complexity. For the computation of the context-based energy, we use an approximated version [63], which is faster than its exact computation for

\begin{tabular}{|c|c|c|}
\hline Module & Parameter & Default value \\
\hline \hline \multirow{2}{*}{ Smoothing } & number of iterations & 10 \\
\cline { 2 - 3 } & diffusion coefficient & 0.14 \\
\hline \multirow{2}{*}{ Get ICC } & Disk's radius for opening & $5 \mathrm{~mm}$ \\
\cline { 2 - 3 } & Thresholding value for ICC & 0.3 \\
\hline Get CSF+Vent & Thresholding value for markers & 0.85 \\
\hline \multirow{2}{*}{ Refine ICC } & Maximal distance defining ICC border & $20 \mathrm{~mm}$ \\
\cline { 2 - 3 } & Maximal closest distance & $2.5 \mathrm{~mm}$ \\
\hline Get BGT & Size of area closing & $0.66 \times$ ICC size \\
\hline \multirow{3}{*}{ Get CoGM } & Horizontal number of blocks & 8 \\
\cline { 2 - 3 } & Vertical number of blocks & 10 \\
\cline { 2 - 3 } & Validation window size & $41 \times 41$ pixels \\
\hline \multirow{2}{*}{ Get WM } & Square size for opening & $1 \times 1 \mathrm{~mm}^{2}$ \\
\hline \multirow{3}{*}{ Get Hyper } & Maximal energy & 0.5 \\
\cline { 2 - 3 } & Parameter $\alpha$ for minimal mean gray level & 1.0 \\
\cline { 2 - 3 } & Minimal relative difference & 0.05 \\
\hline
\end{tabular}

Table 1. Summary of parameters involved in the proposed method. During all our experiments, we have only changed the values of the two parameters in italics.

the extraction of CSF and ventricles. The computation is performed incrementally during the tree construction. For the extraction of hyperintensities, we use an exact computation of the energy [62]. This exact version has a higher time complexity $O\left(n \epsilon^{2} h\right)$, where $h$ is the depth of the maxtree. Yet, it is applied only in the white matter region. So the computation is still rather efficient.

A graphical interface has been developed, integrating each step of the pipeline and a manual correction tool. This tool allows us to improve the segmentation results with little effort by proposing the following operations: 1) remove an entire existing region by selecting an inside point; 2) remove part of an existing region by drawing a closed contour; 3 ) add a region by drawing a closed contour; 4) preserve only a region by drawing a closed contour; 5) preserve only an existing region by choosing an inside point. These manual corrections are easy to perform, are not time consuming, and they help to improve the results.

The graphical interface is illustrated in Figure 12 There are two panels on the top showing the input image and the corresponding results for each processing step. The principle interface is composed of three main blocks: 1) importing images and saving corresponding results; 2) proposed processing pipeline; 3) some manual corrections.

\subsection{Parameter settings}

There are 14 parameters involved in all steps that have been described in the corresponding sections (from 3.2 
to 3.6); their values are listed in Table 1 . The parameter setting for the anisotropic diffusion process, using $1 /\left(1+(\nabla f / K)^{2}\right)$ as the conductance function, is also depicted in Table 1. Most of these parameters are fixed thanks to the prior knowledge about common anatomical characteristics of the neonatal brain and their appearance on the T2-weighted scans. During all our experiments presented in Section 4, we modified only slightly the values of two parameters, $\lambda_{I C C}$ and $\lambda_{m}$ (surrounded by the red rectangles in Figure 12, which are used to extract ICC and markers for CSF. These two parameters have an intuitive meaning with respect to the gray levels of the input image. It is therefore easy to tune them, even for a non-specialist in image processing. The other three parameters related to the hyperintensity extraction were also made available to the users in the graphical interface described previously for the sake of transparency, though we never changed them in any of the experiments. An analysis of the robustness of the parameters is provided in Section 4.5

\section{Experimental results and discussion}

\subsection{Datasets and evaluation measures}

Datasets. The proposed method was tested on images acquired with both $1.5 \mathrm{~T}$ scanners and $3 \mathrm{~T}$ scanners, though it was initially dedicated to $1.5 \mathrm{~T}$ clinical data. To the best of our knowledge, there is no publicly available $1.5 \mathrm{~T}$ neonatal brain MR image dataset providing manual segmentations. Consequently, some experimental results on our in-house clinical $1.5 \mathrm{~T}$ data are presented. First, we tested on 20 supratentorial slices including the basal ganglia and the frontal horns of the periventricular system of 20 axial T2-weighted volumes of preterm newborns, obtained with different 1.5T MR devices. Then we applied the proposed pipeline on three axial T2-weighted volumes (whole image) of three different preterm newborns. Note that these in-house 1.5T MRIs were performed according to the local routine protocol, i.e., without any sedation, and retrospectively studied without the need for internal review board approval. For $3 \mathrm{~T}$ images, we tested on five axial T2-weighted volumes of preterm infants from the NeoBrainS12 dataset [22].

All the tested images were acquired at term-equivalent age (between 39 and 40 weeks of gestation) of preterm

\begin{tabular}{|c|c|c|c|}
\hline Age & 40 weeks & 40 weeks & 40 weeks \\
\hline Protocol & 1.5 T Axial T2 & 1.5 T Axial T2 & 3 T Axial T2 \\
\hline Number of images & 20 & 3 & 5 \\
\hline Rec. matrix & $\begin{array}{c}512 \times 512 \times 20 \text { or } \\
256 \times 256 \times 20\end{array}$ & $\begin{array}{c}512 \times 512 \times 20 \text { or } \\
256 \times 256 \times 20\end{array}$ & $512 \times 512 \times 50$ \\
\hline Voxel sizes $\left(\mathrm{mm}^{3}\right)$ & $\begin{array}{c}0.36 \times 0.36 \times 2.0 \text { to } \\
0.70 \times 0.70 \times 4.0\end{array}$ & $\begin{array}{c}0.39 \times 0.39 \times 5.0 \text { to } \\
0.78 \times 0.78 \times 5.0\end{array}$ & $0.35 \times 0.35 \times 2.0$ \\
\hline TR, TE $(\mathrm{ms})$ & 3750,110 & See Table 3 & 6293,120 \\
\hline
\end{tabular}

Table 2. Acquisition parameters for the images used in this paper.

\begin{tabular}{|c|c|c|c|}
\hline Parameter & Patient 1 & Patient 2 & Patient 3 \\
\hline TR $(\mathrm{ms})$ & 3821 & 3968 & 3747 \\
\hline TE $(\mathrm{ms})$ & 110 & 110 & 110 \\
\hline Rec. matrix & $256 \times 256 \times 20$ & $512 \times 512 \times 18$ & $512 \times 512 \times 17$ \\
\hline Voxel sizes $\left(\mathrm{mm}^{3}\right)$ & $0.78 \times 0.78 \times 5.0$ & $0.39 \times 0.39 \times 5.0$ & $0.39 \times 0.39 \times 5.0$ \\
\hline
\end{tabular}

Table 3. Acquisition parameters for the three patients illustrated in Figure $15 \mathrm{a}-\mathrm{c})$.

infants born at around 28 weeks of gestation. The basic information about these images is listed in Table 2. More details are given in the following.

Slices containing white matter hyperintensities. The first dataset consists of 20 axial T2-weighted supratentorial slices from different 1.5T MR devices (Siemens Avanto, GE Signa Hdxt, Philips Achieva, Philips Intera, Siemens Symphony, Toshiba) of preterm newborns whose clinical status, transfontanellar ultrasound, and electroencephalogram were all normal. White matter hyperintensities may be present in these images. Some examples are shown in Figure 13 a). Two senior observers manually and independently segmented different brain tissues, and detected and segmented white matter hyperintensities.

Three in-house 1.5T T2-weighted volumes. The detailed acquisition parameters for these three patients are listed in Table 3 Two senior observers independently annotated the five tissues for these three volumes. The annotations given by the first observer, who is a more experienced pediatric radiologist than the second one, are used as the reference segmentations. Note that not all pixels are annotated due to the strong partial volume effects and labor intensive work. This corresponds to the usual clinical practice. The interest of the proposed approach is to be reproducible and to provide less un-labeled pixels. The unlabeled pixels are ignored when evaluating the proposed method, i.e., considered as true negative for the five main tissues to be segmented. 
NeoBrainS12 dataset [22]. We have tested the proposed pipeline on the five axial T2-weighted images of preterm infants from the NeoBrainS12 dataset [22]. These images were acquired on a Philips 3T MRI scanner at University Medical Center Utrecht, The Netherlands, with parameters listed in Table 2. On these images, no brain pathology was visible. The manual segmentations were performed either by MDs who were working towards a $\mathrm{PhD}$ in neonatology, or by trained medical students, and further validated by three neonatologists. A detailed description of the data is available at http://neobrains12.isi. $\mathrm{uu} . \mathrm{nl} /$ and in [22]. Note that the NeoBrainS12 dataset also provides two extra axial scans of two preterm infants with reference segmentations for training purpose.

Evaluation measures. Quantitative evaluation of the proposed method on the three datasets described above is based on Dice coefficient (DC), modified (95th percentile) Hausdorff distance (HD), average volume difference (AVD), and mean surface distance (MSD). For an automatic segmentation $S$ and a reference segmentation $G$ of a corresponding tissue, the Dice coefficient is defined as: $D C=2 \times|S \cap G| /(|S|+|G|)$, where $|\cdot|$ denotes the cardinality. The mean surface distance is a contour-based measure which is the mean distance between $\partial S$ and $\partial G$, where $\partial S$ and $\partial G$ denote the set of boundary points of $S$ and respectively $G$.

\subsection{Results on $1.5 T$ clinical images containing white matter hyperintensities}

We tested the proposed pipeline for segmenting neonatal brain tissues including white matter hyperintensities on supratentorial slices of 20 axial T2-weighted volumes obtained with different 1.5T MR scanners (see Section 4.1). The predefined parameters were used. Only a small adaptation was made on the intracranial cavity threshold value (decreased from 0.3 to 0.2 ) for a very few images to precisely segment their ICC. Some boundaries were manually delimited to avoid significant partial volume effect (see Section 4.5 for details about the manual delimitation). The proposed pipeline was applied twice by the same user with a one-month delay. Some qualitative results are depicted in Figure 13. Qualitatively, the segmentation results of the main tissues and the white matter hyperintensities obtained by the proposed method Seg are very close to the manual annotations $G T 1$ and $G T 2$. The differences are better visualized in Figure 13 (e). The violet pixels represent the pixels inside the ICC that the proposed method did not assign with a label of any of the five tissues, but that actually correspond to one of the five tissues. The other colored pixels are the false positives for the corresponding tissue represented by the color. The other gray pixels are the true positives of the corresponding tissue or correct background. Note that we do not consider the segmentation results for the non-annotated pixels, which are considered as true negatives for all the tissues to be segmented.

We have evaluated our results quantitatively based on the Dice coefficient, modified Hausdorff distance, and average volume difference. Table 4 provides the results averaged over all cases for the five main brain tissues, where the extracted white matter hyperintensities are considered as white matter for this evaluation. We compared the results of the two usages described before of the proposed method with potentially different manual interactions denoted as Auto.1 and Auto.2, to the manual segmentation by the senior observers (Obs.). As depicted in this table, the results of the proposed method have generally high Dice coefficients. The fact that this measure is quite sensitive with respect to shape (smaller indices are generally observed on small or thin structures) may explain the smaller values obtained for the gray matter, which is known to be very difficult to observe on such images, in particular due to the strong partial volume effect on thin structures. Note that we do not consider the segmentation results for the non-annotated pixels. Yet, as demonstrated by the relative low Dice coefficient between the two senior observers, the manually labeled pixels do not only contain "easy" pixels. The high Dice coefficients between the two usages of the proposed pipeline with one-month delay by the same user demonstrates the high reproducibility of the proposed semi-automatic method. The same conclusions also hold based on the modified Hausdorff distance and average volume difference. In general, the proposed method performs on par with the two senior observers. For the CSF, the proposed method is more compatible with Obs. 1. Whereas, the proposed method is more consistent with Obs. 2 for the CoGM.

Table 5 provides quantitative results for the DEHSI extraction. Note that the average does not take into account two images (denoted P and Q in Figure 14) in which no hyperintensity is detected by the reference observer 


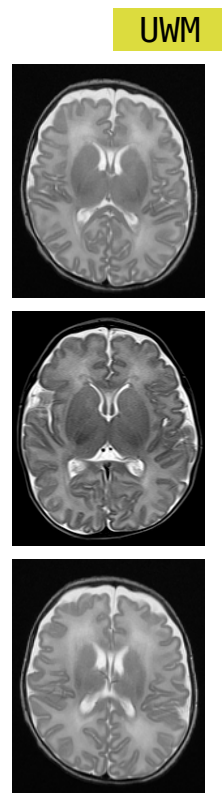

(a) $f$
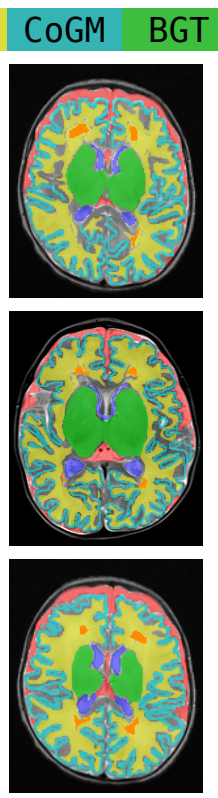

(b) GT\#1
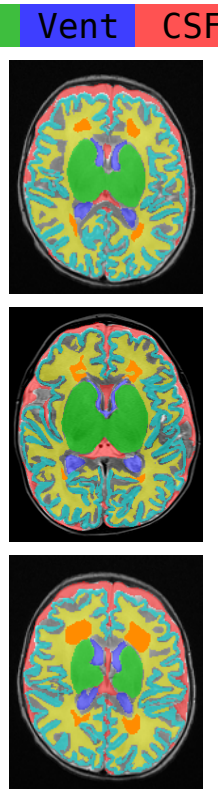

(c) $G T \# 2$
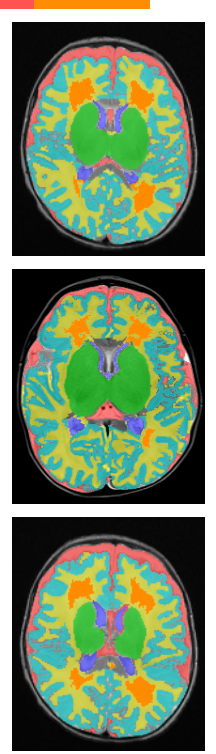

(d) $\mathrm{Seg}$
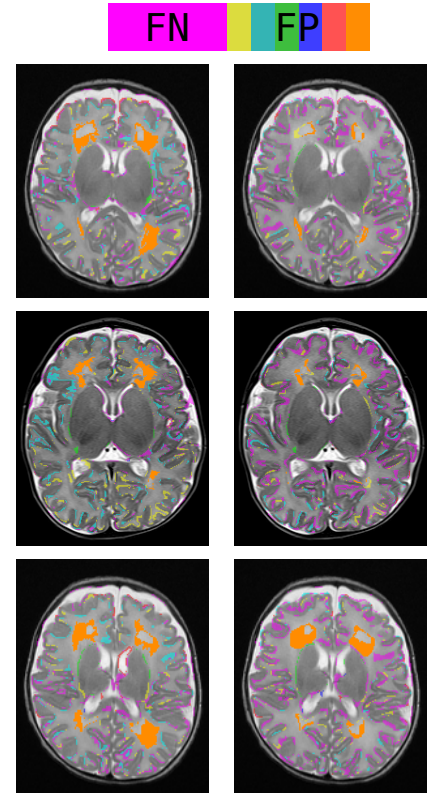

(e) $G T 1 \backslash S e g$

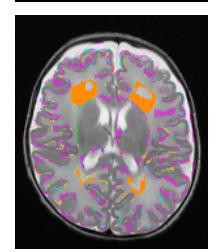

(f) $G T 1 \backslash G T 2$

Figure 13. Some segmentation results using the proposed method on slices of some 1.5T T2-weighted volumes. See the electronic version of this paper for a better visualization.

\begin{tabular}{|c||c|c|c|c|c|c|c||c|c|c||c|c|c|c|c|}
\hline \multicolumn{1}{|c||}{} & \multicolumn{3}{c|}{ UWM } & \multicolumn{3}{c||}{ CoGM } & \multicolumn{3}{c||}{ BGT } & \multicolumn{3}{c||}{ Vent } & \multicolumn{3}{c|}{ CSF } \\
& Dice & HD & AVD & Dice & HD & AVD & Dice & HD & AVD & Dice & HD & AVD & Dice & HD & AVD \\
\hline \hline Obs.1 / Obs.2 & 0.88 & 2.5 & 14.3 & 0.78 & 2.1 & 17.7 & 0.97 & 1.5 & 02.7 & 0.94 & 0.9 & 07.5 & 0.88 & 1.6 & 16.1 \\
\hline Obs.1 / Auto.1 & 0.86 & 2.8 & 10.1 & 0.79 & 2.6 & 09.9 & 0.96 & 1.7 & 02.7 & 0.89 & 1.7 & 17.1 & 0.88 & 3.6 & 13.5 \\
\hline Obs.2 / Auto.1 & 0.88 & 3.9 & 05.4 & 0.86 & 1.8 & 08.8 & 0.96 & 1.6 & 04.3 & 0.88 & 1.8 & 18.0 & 0.83 & 8.8 & 16.1 \\
\hline Auto.1 / Auto.2 & 0.97 & 1.5 & 02.4 & 0.98 & 0.4 & 02.1 & 0.99 & 0.6 & 01.0 & 0.98 & 0.3 & 02.3 & 0.98 & 5.7 & 04.0 \\
\hline
\end{tabular}

Table 4. Mean Dice coefficients, modified Hausdorff distances (HD), and average volume difference (AVD) between senior observers and two usages of the proposed semi-automatic method on the 20 images that potentially contain DEHSI.

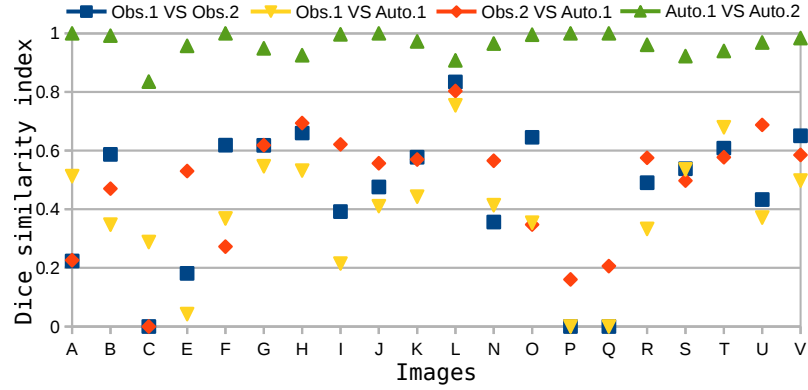

Figure 14. Evaluation of Dice coefficient of DEHSI segmentation on 20 images.
Obs.1. As shown in [33], deciding visually whether hyperintensities are present or not is highly subjective, and the precise delineation of the corresponding regions is even more difficult. This is confirmed by the low values of Dice coefficient, and high values of HD and AVD. Yet, the variability of the proposed method with respect to observers is of the same order as the variability between observers (Obs.1 and Obs.2), and the proposed method remains very reproducible, which is an important improvement over manual segmentation. A detailed quantitative evaluation based on Dice coefficient for each of the $20 \mathrm{im}$ ages is depicted in Figure 14. Note that if there were no hyperintensity marked in one segmentation result, but detected in another segmentation result, the Dice coefficient 


\begin{tabular}{|c|c|c||c|c|c||c|c|c||c|c|c|}
\hline \multicolumn{3}{|c||}{ Obs.1 / Obs.2 } & \multicolumn{3}{c||}{ Obs.1 / Auto.1 } & \multicolumn{3}{c||}{ Obs.2 / Auto.1 } & \multicolumn{3}{c|}{ Auto.1 / Auto.2 } \\
Dice & HD & AVD & Dice & HD & AVD & Dice & HD & AVD & Dice & HD & AVD \\
\hline 0.49 & 22.5 & 132.6 & 0.42 & 22.5 & 244.7 & 0.51 & 27.3 & 78.3 & 0.96 & 7.2 & 8.2 \\
\hline
\end{tabular}

Table 5. Mean Dice coefficients, modified Hausdorff distances (HD), and average volume difference (AVD) for the DEHSI segmentation on 18 images.

between these two results would be 0 (e.g., for images $C$, $\mathrm{P}$, and Q).

\subsection{Results on clinical images obtained with 1.5T MRI}

The proposed pipeline was tested on three in-house 1.5T T2-weighted volumes described in Section 4.1. Some results can be compared qualitatively to the two manual annotations GT1 and GT2 in Figure 15 As illustrated in this figure (better visualized in the difference images on the right side), the results of the proposed method Seg are very similar to the manual annotations. Note that the pixels that are not annotated by the reference radiologist are ignored when computing the segmentation differences. The inter-individual variabilities between the two observers, and the inter-individual variabilities between our results and the reference observer's annotations are comparable.

Quantitative analyses on these three patients (whole brains) are depicted in Table 6. The Dice coefficients are slightly worse compared to the previous experiment. With the whole exploration of the supratentorial stage, the partial volume effect and the complexity of the segmentation could explain this slight decrease. Yet, our results are comparable with the manual annotations. Note that the pixels not annotated by the reference pediatric radiologist are ignored for this evaluation, i.e., considered as true negative for the five main tissues to be segmented. However, as depicted by the relative low values of Dice coefficient between the two senior observers, the evaluation is not only performed on the "easy" voxels. Three usages of the proposed pipeline by the same user with different levels of manual segmentations are evaluated in Table 6 1) segmentation without any manual corrections denoted by AutoMin; 2) segmentation with very few manual corrections that are easy to apply, denoted by Auto (e.g., Figure 15; 3) segmentation with manual corrections to achieve a very precise segmentation denoted by AutoMax (see Section 4.5 for details about the manual corrections). As depicted in Table 6, the proposed pipeline is quite robust even if no manual corrections are applied. The same

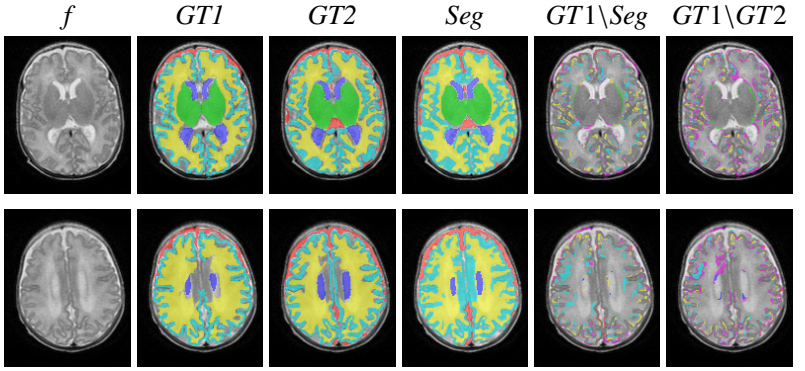

(a) On slices of the $1.5 \mathrm{~T}$ volume of patient 1 .

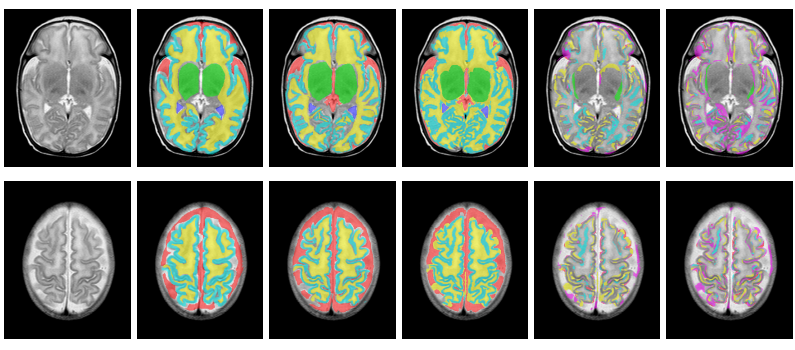

(b) On slices of the $1.5 \mathrm{~T}$ volume of patient 2.
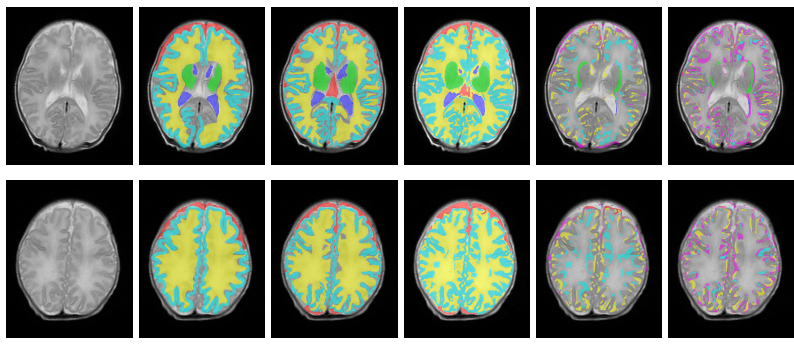

(c) On slices of $1.5 \mathrm{~T}$ volume of patient 3.

Figure 15. Some segmentation results using the proposed method on slices of three 1.5T T2-weighted volumes. See the electronic version of this paper for a better visualization.

conclusions also hold based on the evaluation with HD and AVD. The provided ordinary manual corrections allow us to improve easily the segmentation accuracy.

We have attempted to compare the proposed method with other state-of-the-art methods (mostly dedicated for 


\begin{tabular}{|c|c|c|c|c|c|c|c|c|c|c|c|c|c|c|c|c|}
\hline \multicolumn{2}{|r|}{ Patient } & \multicolumn{2}{|c|}{$\begin{array}{r}\mathbf{U W 1} \\
\text { Dice } \mid \mathrm{HD}\end{array}$} & $\begin{array}{l}\mathbf{M} \\
\mathrm{D} \mid \mathrm{AVD}\end{array}$ & & \multicolumn{2}{|c|}{ OGM } & & BGT & & \multicolumn{3}{|c|}{ Vent } & \multicolumn{2}{|c|}{ CSF } & \\
\hline \multirow{4}{*}{ \#1 } & Obs.1/Obs.2 & 0.85 & 2.0 & 112.6 & 0.73 & 2.0 & 26.8 & 0.95 & 2.0 & 02.3 & 0.87 & 7.9 & 12.2 & 0.87 & 2.0 & 12.7 \\
\hline & Obs.1 / AutoMin & 0.85 & 3.2 & 02.3 & 0.71 & 4.0 & 08.8 & 0.93 & 3.0 & 07.7 & .88 & 2.0 & 20.3 & 0.83 & 5.1 & \\
\hline & Obs.1/Auto & 0.86 & 3.0 & 03.1 & 0.72 & 4.0 & 07.8 & 0.94 & 2.0 & .2 & 88 & 2.0 & 19.6 & 0.84 & 5.0 & 11.1 \\
\hline & Obs.1/A & 0.87 & 2.2 & 01.4 & 0.73 & 2.8 & 13.6 & 0.95 & 2.0 & 02.5 & 0.90 & 1.4 & 16.1 & 0.84 & 3.6 & 10.5 \\
\hline \multirow{4}{*}{ \#2 } & & & 5.0 & 06.7 & 66 & 4.1 & 33.1 & 0.96 & 4.0 & 0.4 & 91 & 2.8 & 8.6 & 0.86 & 6.1 & 11.4 \\
\hline & Obs. & 0.75 & 6.4 & 05.6 & 0.65 & 5.1 & 02.0 & 0.94 & 5.7 & 09.7 & .73 & 14.6 & 37.3 & 0.80 & 5.7 & 18.7 \\
\hline & & 0.76 & 6.2 & 05.7 & 0.64 & 5.1 & 00.2 & 0.94 & 4.6 & 00.2 & 0.73 & 17.1 & 38.2 & 0.81 & 7.0 & 15.3 \\
\hline & Obs. & 0.81 & 5.5 & 5.5 & .66 & 5.0 & 08.1 & 0.96 & 4.0 & 02.1 & 79 & 15. & 32.4 & 0.81 & 10.8 & 12.4 \\
\hline \multirow{4}{*}{ \#3 } & Obs.1 / Obs.2 & 0.80 & 5.2 & 08.4 & 0.64 & 4.5 & 37.9 & 0.91 & 6.7 & 08.7 & 88 & 9.8 & 01.1 & 0.7 & 29. & 21.4 \\
\hline & Obs.1/AutoMin & 0.75 & 7.1 & 02.8 & 0.67 & 5.5 & 10.4 & 0.92 & 5.1 & 12.8 & 80 & 4.5 & 25.0 & 0.68 & 34.7 & $20 .($ \\
\hline & & 0.76 & 6.5 & 01.4 & 0.67 & 5.5 & 09.0 & 0.93 & 4.2 & 05.7 & 79 & 5.0 & 25.8 & 0.72 & 19.2 & 21.7 \\
\hline & Obs.1 & 0.82 & 5.7 & 04.4 & 0.69 & 4.5 & 19.9 & 0.96 & 4.1 & 01.0 & 0.84 & 5.0 & 16.0 & 0.72 & 36.0 & 13.7 \\
\hline \multirow{4}{*}{ 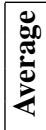 } & $\mathrm{Obs}$ & & 4.0 & 097 & & 35 & 326 & 0.94 & 4.2 & 03.8 & & & 073 & 0.82 & 12 & 151 \\
\hline & Obs. & 0. & 5 & & & & & & & & & & & & & \\
\hline & & & 5. & 03 & & 49 & & 0.94 & 3. & 02 & & & 27 & 0.79 & 10.4 & 16.0 \\
\hline & Obs.1 & 0.83 & 4.5 & 4.9 & & 4.1 & & & & & & 7.2 & 21. & 0.79 & 16. & 12. \\
\hline
\end{tabular}

Table 6. Mean Dice coefficients, modified Hausdorff distances (HD), and average volume difference (AVD) between senior observers and the proposed method with different level of manual corrections on $1.5 \mathrm{~T} \mathrm{~T} 2$ weighted volumes of three patients. See the corresponding text for details.

3T images) on these $1.5 \mathrm{~T}$ images, we only found one publicly available source code in [28] which is dedicated to $3 \mathrm{~T}$ images. It works very poorly on these $1.5 \mathrm{~T}$ images. Consequently, no qualitative nor quantitative comparison is given on these $1.5 \mathrm{~T}$ images.

\subsection{Results on images obtained with $3 T$ MRI}

We also tested our method on 3T axial images in the NeoBrainS12 dataset [22] to illustrate the generalization power of the proposed approach. These data have not exhibited white matter hyperintensities, but our approach achieved very accurate segmentation results, depicted in Figure 16 Please note that the black pixels inside the ICC of manual segmentations in the second row of Figure 16 are myelinated white matter (MWM) that we do not segment. For our segmentation results, not all the pixels inside the ICC were assigned to a tissue. The differences between our segmentation results and the reference were highlighted on the bottom of Figure 16 Figure 17 i1lustrates some segmentation results given by the proposed method on three preterm infants in the test dataset of NeoBrainS12 [22]. It should be mentioned that, in daily practice, no isotropic scan is performed on 1.5T acquisition systems, since the age of the patients requires very short acquisition time. Although all steps of the method apply in 3D as well, such an extension was not tested because of
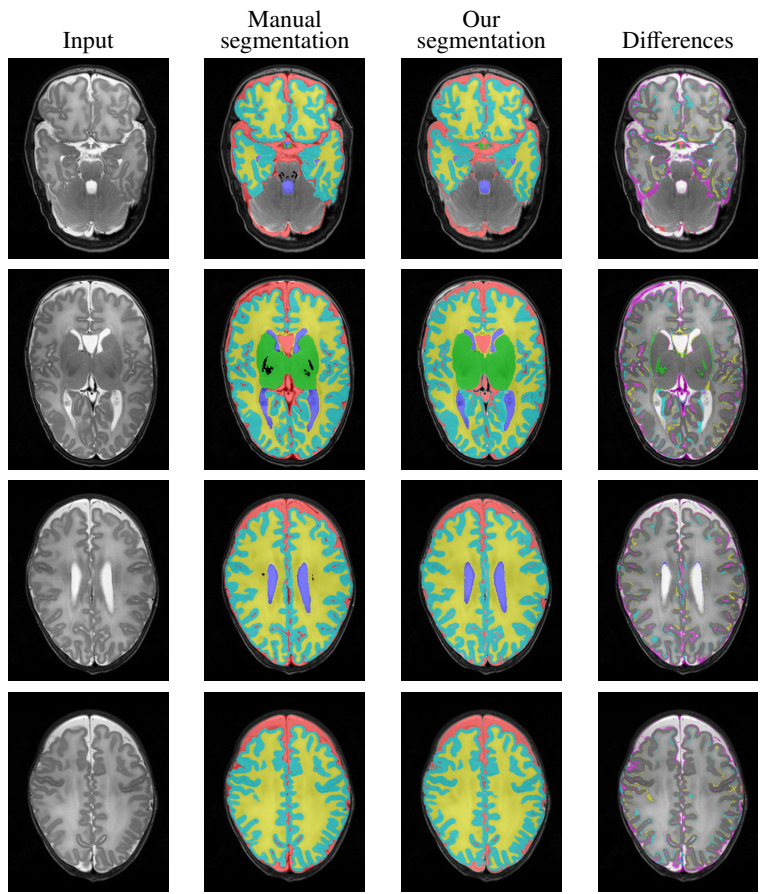

Figure 16. Some segmentation results using the proposed method on several slices of the T2-weighted volume for a patient in the training set of NeoBrainS12 dataset [22]. See the electronic version of this paper for a better visualization. 


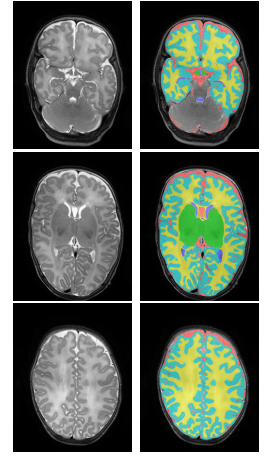

(a) Set_i1

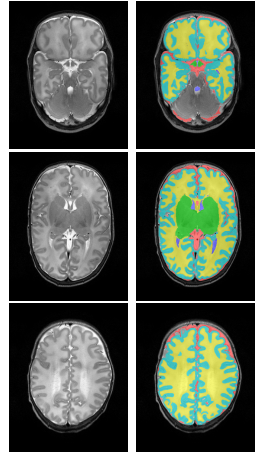

(b) Set_i2

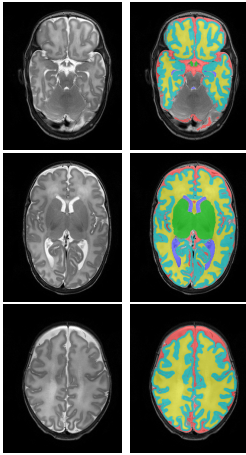

(c) Set_i3

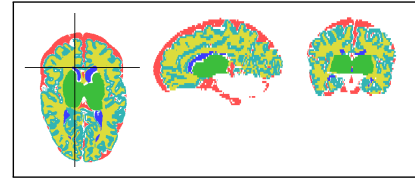

(d) Out-of-plane views of a segmentation

Figure 17. (a-c): Segmentation results using the proposed pipeline on slices of T2-weighted volumes for three preterm infants from the NeoBrainS12 dataset [22]; each column corresponds to 3 slices of the same subject and their corresponding segmentations by the proposed method (d): Although the pipeline processes the slices independently, the final $3 \mathrm{D}$ result is spatially consistent.

the lack of data. Noteworthy, the proposed method generalizes well to the NeoBrains 12 data test set, although it was not specifically designed for it, alluding to the ability of the method in its current state to deal with isotropic scans. The 3D spatial consistency on out-of-plane slices is illustrated in Figure 17 Let us remark that there is no topological constraint in the method and, as a consequence, the final topology of the extracted structures is not guaranteed. Yet, the limited spatial resolution does not allow for a rigorous 3D topological analysis; furthermore, the intended applications require merely volumetric analysis, where topology does not play an important role.

The comparison with other state-of-the-art methods on this dataset is presented in Table 7. Note that the competitive method UNC-IDEA [54] is a learning-based approach that makes use of a probability map for different tissues estimated by other learning-based approaches. With the exception of DCU, all the other methods are based on an atlas created from other datasets and/or improved by the two provided training volumes. As shown in Table 7, the proposed method achieves results comparable to the state-of-the-art methods.

\subsection{Discussion}

These experimental results demonstrate the potential of the proposed method for segmenting neonatal brain structures, particularly for automatic DEHSI segmentation for neonates that has never been addressed. The high reproducibility of the algorithm is an important improvement over existing visual DEHSI assessment methods. It will thereby constitute a powerful tool prior to any clinical study for correlations and thus a better understanding of the prognostic value for DEHSI. Though the proposed method was originally developed for 1.5T MRI data from usual clinical practice, it performs equally well for 3T MRI data that are likely to be more and more developed in clinical practice. As compared to the classical atlas-based methods that may require different atlases and registration processes for different volumes, the proposed method based on their common anatomical characteristics and appearance on T2-weighted MR images is more generic.

For all the experiments in this paper, we set 12 of 14 involved parameters thanks to the prior common knowledge about the corresponding structures. We only changed the value for the two parameters $\lambda_{I C C}$ and $\lambda_{m}$ that are used to extract ICC and respectively CSF markers. These two parameters have an intuitive meaning with respect to the gray levels of the input image. Consequently, they are easy to tune, even for a non-specialist in image processing. Besides, we have changed their values at most twice for each volume. Furthermore, even though these two parameters may have slight impact on the extraction of ICC and CSF, leading to approximately correct segmentation for the other structures, they do not influence the segmentation of white matter hyperintensities.

The proposed segmentation pipeline is a semiautomatic method. It requires two types of human interventions: 1) Setting several inside (and outside if necessary) ventricle markers for each slice containing ventricles; 2) Setting two inside BGT markers and a BGT constraint rectangle for each slice containing BGT. The bounding rectangle has to be delimited approximatively around the basal ganglia to limit the area of segmentation, which is an easy task, that furthermore does not need to be very precise. Note that a marker is a point, so these 


\begin{tabular}{|c||c|c||c|c||c|c||c|c||c|c|}
\hline \multirow{2}{*}{ Method } & \multicolumn{2}{|c||}{ UWM } & \multicolumn{2}{c|}{ CoGM } & \multicolumn{2}{c||}{ BGT } & \multicolumn{2}{c|}{ Vent } & \multicolumn{2}{c|}{ CSF } \\
& DC & MSD & DC & MSD & DC & MSD & DC & MSD & DC & MSD \\
\hline \hline DCU & 0.83 & 0.40 & - & - & - & - & - & - & - & - \\
\hline DTC [56] & 0.89 & 0.22 & 0.84 & 0.16 & 0.88 & $\mathbf{0 . 4 7}$ & $\mathbf{0 . 8 5}$ & $\mathbf{0 . 2 2}$ & $\mathbf{0 . 7 6}$ & 0.35 \\
\hline Imperial [28] & $\mathbf{0 . 9 0}$ & 0.18 & 0.85 & 0.17 & 0.90 & 0.62 & 0.81 & 1.38 & $\mathbf{0 . 7 9}$ & $\mathbf{0 . 3 2}$ \\
\hline MCRI & 0.88 & 0.25 & 0.84 & 0.19 & 0.88 & 0.70 & 0.77 & 0.35 & 0.73 & 0.56 \\
\hline UCL [29] & 0.87 & 0.26 & 0.83 & 0.18 & 0.89 & 0.56 & 0.81 & 0.32 & 0.71 & 0.54 \\
\hline UNC-IDEA [54] & $\mathbf{0 . 9 2}$ & $\mathbf{0 . 1 3}$ & $\mathbf{0 . 8 6}$ & $\mathbf{0 . 1 1}$ & $\mathbf{0 . 9 2}$ & $\mathbf{0 . 3 3}$ & 0.79 & 0.25 & $\mathbf{0 . 7 9}$ & $\mathbf{0 . 2 5}$ \\
\hline UPenn [61] & 0.85 & 0.38 & 0.80 & 0.27 & 0.80 & 1.25 & $\mathbf{0 . 8 6}$ & $\mathbf{0 . 2 2}$ & 0.61 & 0.74 \\
\hline Our & $\mathbf{0 . 9 2}$ & $\mathbf{0 . 1 4}$ & $\mathbf{0 . 8 7}$ & $\mathbf{0 . 1 3}$ & $\mathbf{0 . 9 1}$ & 0.62 & 0.83 & $\mathbf{0 . 2 4}$ & 0.68 & 0.61 \\
\hline
\end{tabular}

Table 7. Quantitative comparison of different methods on the NeoBrainS12 dataset [22]. Note that the numbers in light gray for methods MCRI and UNC-IDEA [54] are evaluation results for CSF+Ventricles. The methods using more training images than those provided in this dataset are not included.

settings require around 10 clicks only, which was found acceptable by the clinicians. These interventions could be removed in the future by investigating automatic settings based on their relative positions. To achieve very precise segmentations, we have applied some simple manual corrections summarized as follows: 1) Drawing a region to complete the extracted ICC, if necessary (less than 20 times for all experiments); 2) Adding inside markers for some missed small CSF regions by clicking inside them (less than 10 clicks for each slice); 3) Delimiting boundaries of BGT by drawing regions to be removed; 4) Delimiting some boundaries of CoGM by cutting several times (i.e., drawing regions to be removed) to isolate it from the other tissues (e.g., cerebellum), followed by several clicks to extract the expected CoGM; 5) Clicking several times inside the expected UWM to extract it, and if necessary (less than 20 times for all experiments), delimiting some boundaries of UWM to isolate it from other tissues before clicking. These corrections, that do not require further precise anatomical knowledge, are easy to apply and are not time consuming; they only take a dozen of seconds. Note that, from our experiences, the ratio between subjects with and without correction has been $1 / 3$ (for example, a required correction concerned the exclusion of pellucidum cyst). Yet, as depicted in Table 6 , the proposed semi-automatic pipeline is also robust even if no manual corrections are applied.

Although the proposed pipeline required some user interactions (discussed above), results were reproducible and robust. Semi-automation is even an advantage since the functionalities offered to the users, such as defining some markers manually and carrying out some manual corrections, were in adequation with the increasing need for providing medical experts with semi-automatic analysis tools. In particular, this allows obtaining results that fit each specific clinical requirement. Moreover, semiautomation makes the method more general and adaptable: there is no prior information needed about the orientation of the brain MRI, and the method could be adapted to other pathologies (such as ventricular dilatation). This will be further demonstrated in our future works.

Note also that the intended use of the method is in clinical applications, where the data of each subject will be processed just after their MRI acquisitions.

The proposed pipeline enabled the precise segmentation of a volume slice in approximately 2 minutes. This was considerably faster than a manual annotation, which could take 45 minutes on average to produce similar segmentation results for each slice. As compared to the classical atlas-based methods, which usually involve a timeconsuming registration process (e.g., the method in [28] takes about 5 hours to segment a $1.5 \mathrm{~T}$ volume with 20 slices), the processing time for the proposed method is acceptable.

Feedback from radiologists is very positive: they could use the program very easily in daily practice, without changing any parameters in almost all tested cases. They found the graphical interface easy to understand and to use. They also found it convenient to be able to check each step of each segmentation result, which is displayed immediately. It allows to correct one step if needed, thus preventing an incorrect result to have an impact on the subsequent ones. Besides, although fully automatic methods can provide accurate segmentation in some cases, the radiologists usually have to correct the segmentations according to their own purposes. The proposed semi- 


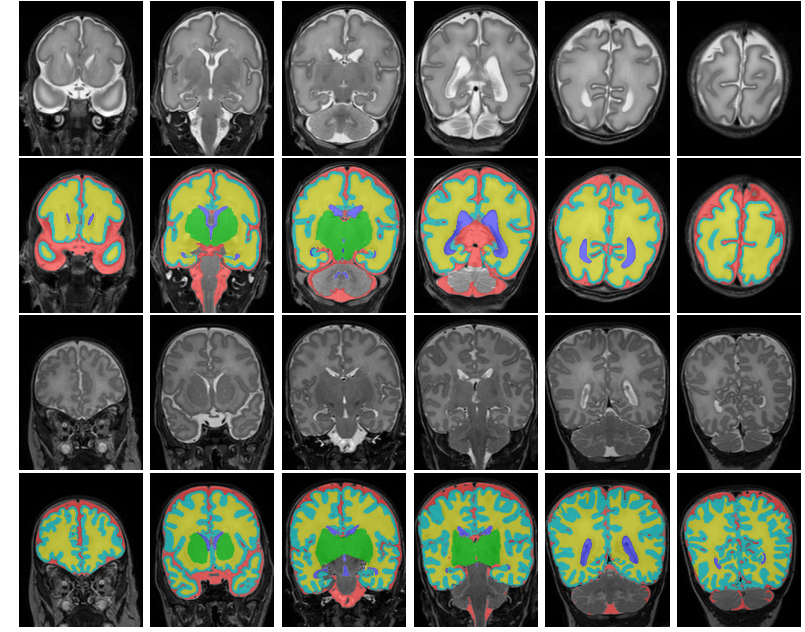

Figure 18. Preliminary segmentation results on several slices of two coronal T2-weighted MR images of two infants acquired at 30 weeks (top two rows) and respectively 40 weeks (bottom two rows) corrected gestational age from NeoBrainS12 dataset [22]

automatic method is a good compromise between efficiency and generality, and is in increasing need for radiologists in practice. Indeed, prognostic and diagnostic clinical value of DEHSI is unknown yet as it would require a follow-up of several years to evaluate the neurocognitive functions of the subjects. Though such clinical data are not available at this point, the proposed method provides a powerful tool to achieve such follow-up, explaining the effect of DEHSI.

\section{Conclusion}

A complete segmentation pipeline for neonatal brain T2-weighted MR images based on an original exploitation of max-tree image representations was proposed. Although the individual components of the method rely indeed on existing methods and tools, the main novelty of the method relies in the proposed pipeline for DEHSI detection, in its adaptation to the specific anatomy of neonates brains, in the absence of critical parameters to tune, and in the compromise between automated steps and a reasonable level of control left to the user. The automatic and reproducible detection of DEHSI of the white matter should allow for improved analysis of MR images.
It could lead to a better understanding of the DEHSI and improve medical care for premature newborns. The high reproducibility of the algorithm is an important improvement over existing visual assessment methods. The proposed method is currently tested on images of premature newborns acquired in different hospitals, which could lead to a future study of its potential use in multi-centric studies. Furthermore, a preliminary evaluation on 3T MRI data confirmed the versatility of our approach, to process images of variable quality and acquired under various conditions. This was an important result, since 3T MRI is likely to be more and more developed in clinical practice. The algorithm, along with its efficient implementation and user-friendly interface, will thereby constitute a powerful tool with useful clinical applications.

A major perspective is to adapt the proposed pipeline to the coronal slices. Indeed, axial and coronal slices feature similar anatomical characteristics used in the proposed pipeline. Consequently, the adaptation would be straightforward. Some preliminary results on the coronal images from NeoBrainS12 dataset [22] are illustrated in Figure 18 .

\section{Acknowledgments}

This work has been done during the $\mathrm{PhD}$ thesis of Baptiste Morel at LTCI, Télécom ParisTech, and at KremlinBicêtre Hospital, and during the post-doctoral work of Sonia Dahdouh at LTCI, Télécom ParisTech (partially funded by a grant from ANSES). The authors are grateful to John C. Scatarige and Anne Trager for English language assistance, and to Trousseau Hospital, Paris, for providing some of the data used in the evaluation of this work.

\section{References}

[1] Admiraal-Behloul, F., Van Den Heuvel, D., Olofsen, H., van Osch, M., van der Grond, J., Van Buchem, M., Reiber, J., 2005. Fully automatic segmentation of white matter hyperintensities in MR images of the elderly. NeuroImage 28, 607-617.

[2] Anbeek, P., Išgum, I., van Kooij, B., Mol, C.P., Kersbergen, K.J., Groenendaal, F., Viergever, M.A., 
de Vries, L.S., Benders, M., 2013. Automatic segmentation of eight tissue classes in neonatal brain MRI. PloS one 8, e81895.

[3] Anbeek, P., Vincken, K.L., Van Osch, M., Bisschops, R., Van Der Grond, J., 2004. Probabilistic segmentation of white matter lesions in MR imaging. NeuroImage 21, 1037-1044.

[4] Beare, R.J., Chen, J., Kelly, C.E., Alexopoulos, D., Smyser, C.D., Rogers, C.E., Loh, W.Y., Matthews, L.G., Cheong, J.L.Y., Spittle, A.J., Anderson, P.J., Doyle, L.W., Inder, T.E., Seal, M.L., Thompson, D.K., 2016. Neonatal brain tissue classification with morphological adaptation and unified segmentation. Frontiers in Neuroinformatics 10, 1-17.

[5] Berger, C., Géraud, T., Levillain, R., Widynski, N., Baillard, A., Bertin, E., 2007. Effective component tree computation with application to pattern recognition in astronomical imaging, in: IEEE International Conference on Image Processing, pp. 41-44.

[6] de Bruïne, F.T., van den Berg-Huysmans, A.A., Leijser, L.M., Rijken, M., Steggerda, S.J., van der Grond, J., van Wezel-Meijler, G., 2011. Clinical implications of MR imaging findings in the white matter in very preterm infants: a 2-year follow-up study. Radiology 261, 899-906.

[7] Caligiuri, M.E., Perrotta, P., Augimeri, A., Rocca, F., Quattrone, A., Cherubini, A., 2015. Automatic detection of white matter hyperintensities in healthy aging and pathology using magnetic resonance imaging: A review. Neuroinformatics 13, 261-276.

[8] Cardoso, M.J., Melbourne, A., Kendall, G.S., Modat, M., Robertson, N.J., Marlow, N., Ourselin, S., 2013. AdaPT: an adaptive preterm segmentation algorithm for neonatal brain MRI. NeuroImage 65, 97-108.

[9] Carlier, G., Comte, M., Peyré, G., 2009. Approximation of maximal Cheeger sets by projection. ESAIM: Mathematical Modelling and Numerical Analysis 43, 139-150.
[10] Carlinet, E., Géraud, T., 2014. A comparative review of component tree computation algorithms. IEEE Transactions on Image Processing 23, 3885-3895.

[11] Cheong, J.L., Thompson, D.K., Spittle, A.J., Potter, C.R., Walsh, J.M., Burnett, A.C., Lee, K.J., Chen, J., Beare, R., Matthews, L.G., et al., 2016. Brain volumes at term-equivalent age are associated with 2 year neurodevelopment in moderate and late preterm children. The Journal of pediatrics 174, 91-97.e1.

[12] Counsell, S.J., Allsop, J.M., Harrison, M.C., Larkman, D.J., Kennea, N.L., Kapellou, O., Cowan, F.M., Hajnal, J.V., Edwards, A.D., Rutherford, M.A., 2003. Diffusion-weighted imaging of the brain in preterm infants with focal and diffuse white matter abnormality. Pediatrics 112, 1-7.

[13] Counsell, S.J., Maalouf, E.F., Fletcher, A.M., Duggan, P., Battin, M., Lewis, H.J., Herlihy, A.H., Edwards, A.D., Bydder, G.M., Rutherford, M.A., 2002. MR imaging assessment of myelination in the very preterm brain. American Journal of Neuroradiology $23,872-881$.

[14] Despotović, I., Goossens, B., Philips, W., 2015. MRI segmentation of the human brain: challenges, methods, and applications. Computational and Mathematical Methods in Medicine 2015. doi 10 . $1155 / 2015 / 450341$

[15] Devi, C.N., Chandrasekharan, A., Sundararaman, V., Alex, Z.C., 2015. Neonatal brain MRI segmentation: A review. Computers in Biology and Medicine 64, 163-178.

[16] Dubois, J., Germanaud, D., Angleys, H., et al., 2016. Exploring the successive waves of cortical folding in the developing brain using MRI and spectral analysis of gyrification, in: IEEE International Symposium on Biomedical Imaging (ISBI), pp. 261-264.

[17] Garel, C., Chantrel, E., Elmaleh, M., Brisse, H., Sebag, G., 2003. Fetal MRI: normal gestational landmarks for cerebral biometry, gyration and myelination. Child's Nervous System 19, 422-425.

[18] Gousias, I.S., Edwards, A.D., Rutherford, M.A., Counsell, S.J., Hajnal, J.V., Rueckert, D., Hammers, 
A., 2012. Magnetic resonance imaging of the newborn brain: manual segmentation of labelled atlases in term-born and preterm infants. NeuroImage 62, 1499-1509.

[19] Griffanti, L., Zamboni, G., Khan, A., Li, L., Bonifacio, G., Sundaresan, V., Schulz, U.G., Kuker, W., Battaglini, M., Rothwell, P.M., et al., 2016. BIANCA (brain intensity abnormality classification algorithm): A new tool for automated segmentation of white matter hyperintensities. NeuroImage 141, 191-205.

[20] Gui, L., Lisowski, R., Faundez, T., Hüppi, P.S., Lazeyras, F., Kocher, M., 2012. Morphology-driven automatic segmentation of MR images of the neonatal brain. Medical Image Analysis 16, 1565-1579.

[21] Guigues, L., Cocquerez, J.P., Le Men, H., 2006. Scale-sets image analysis. International Journal of Computer Vision 68, 289-317.

[22] Išgum, I., Benders, M.J., Avants, B., Cardoso, M.J., Counsell, S.J., Gomez, E.F., Gui, L., Húppi, P.S., Kersbergen, K.J., Makropoulos, A., et al., 2015. Evaluation of automatic neonatal brain segmentation algorithms: the NeoBrainS12 challenge. Medical Image Analysis 20, 135-151.

[23] Ismail, M., Soliman, A., ElTanboly, A., Switala, A., Mahmoud, M., Khalifa, F., Gimel'farb, G., Casanova, M.F., Keynton, R., El-Baz, A., 2016. Detection of white matter abnormalities in MR brain images for diagnosis of autism in children, in: IEEE 13th International Symposium on Biomedical Imaging (ISBI), pp. 6-9.

[24] Jeon, T.Y., Kim, J.H., Yoo, S.Y., Eo, H., Kwon, J.Y., Lee, J., Lee, M., Chang, Y.S., Park, W.S., 2012. Neurodevelopmental outcomes in preterm infants: comparison of infants with and without diffuse excessive high signal intensity on MR images at nearterm-equivalent age. Radiology 263, 518-526.

[25] Klöppel, S., Abdulkadir, A., Hadjidemetriou, S., Issleib, S., Frings, L., Thanh, T.N., Mader, I., Teipel, S.J., Hüll, M., Ronneberger, O., 2011. A comparison of different automated methods for the detection of white matter lesions in MRI data. NeuroImage $57,416-422$.

[26] Klöppel, S., Stonnington, C.M., Chu, C., Draganski, B., Scahill, R.I., Rohrer, J.D., Fox, N.C., Jack, C.R., Ashburner, J., Frackowiak, R., 2008. Automatic classification of MR scans in Alzheimer's disease. Brain 131, 681-689.

[27] Makropoulos, A., Counsell, S.J., Rueckert, D., 2017. A review on automatic fetal and neonatal brain MRI segmentation. NeuroImage , 1-18In press.

[28] Makropoulos, A., Gousias, I.S., Ledig, C., Aljabar, P., Serag, A., Hajnal, J.V., Edwards, A.D., Counsell, S.J., Rueckert, D., 2014. Automatic whole brain MRI segmentation of the developing neonatal brain. IEEE Transactions on Medical Imaging 33, 1818 1831.

[29] Melbourne, A., Cardoso, M.J., Kendall, G.S., Robertson, N.J., Marlow, N., Ourselin, S., 2012. NeoBrainS12 challenge: Adaptive neonatal MRI brain segmentation with myelinated white matter class and automated extraction of ventricles I-IV. MICCAI Grand Challenge: Neonatal Brain Segmentation (NeoBrainS12), 16-21.

[30] Mewes, A.U.J., Hüppi, P.S., Als, H., Rybicki, F.J., Inder, T.E., McAnulty, G.B., Mulkern, R.V., Robertson, R.L., Rivkin, M.J., Warfield, S.K., 2006. Regional brain development in serial magnetic resonance imaging of low-risk preterm infants. Pediatrics 118, 23-33.

[31] Moeskops, P., Benders, M.J., Chit, S.M., Kersbergen, K.J., Groenendaal, F., de Vries, L.S., Viergever, M.A., Išgum, I., 2015. Automatic segmentation of MR brain images of preterm infants using supervised classification. NeuroImage 118, 628-641.

[32] Moeskops, P., Viergever, M.A., Mendrik, A.M., de Vries, L.S., Benders, M., Išgum, I., 2016. Automatic segmentation of MR brain images with a convolutional neural network. IEEE Transactions on Medical Imaging 35, 1252-1261. 
[33] Morel, B., Antoni, G., Teglas, J., Bloch, I., Adamsbaum, C., 2016. Neonatal brain MRI: how reliable is the radiologist's eye? Neuroradiology 58, 189-193.

[34] Morel, B., Xu, Y., Virzi, A., Géraud, T., Adamsbaum, C., Bloch, I., 2016. A challenging issue: detection of white matter hyperintensities in neonatal brain MRI, in: International Conference of the IEEE Engineering in Medicine and Biology Society (EMBC), Orlando, FL, USA. pp. 93-96.

[35] Mumford, D., Shah, J., 1989. Optimal approximations by piecewise smooth functions and associated variational problems. Communications on Pure and Applied Mathematics 42, 577-685.

[36] Najman, L., Couprie, M., 2006. Building the component tree in quasi-linear time. IEEE Transactions on Image Processing 15, 3531-3539.

[37] Otsu, N., 1979. A threshold selection method from gray-level histograms. IEEE Transactions on Systems, Man and Cybernetics 9, 62-66.

[38] Parazzini, C., Righini, A., Rustico, M., Consonni, D., Triulzi, F., 2008. Prenatal magnetic resonance imaging: brain normal linear biometric values below 24 gestational weeks. Neuroradiology 50, 877-883.

[39] Parikh, N.A., Lasky, R.E., Kennedy, K.A., McDavid, G., Tyson, J.E., 2013. Perinatal factors and regional brain volume abnormalities at term in a cohort of extremely low birth weight infants. Plos One 8 , e62804.

[40] Parikh, N.A., Pierson, C.R., Rusin, J.A., 2016. Neuropathology associated with diffuse excessive high signal intensity abnormalities on MRI in very preterm infants. Pediatric Neurology , In Press, DOI: 10.1016/j.pediatrneurol.2016.07.006.

[41] Perona, P., Malik, J., 1990. Scale-space and edge detection using anisotropic diffusion. IEEE Transactions on Pattern Analysis and Machine Intelligence 12, 629-639.

[42] Prastawa, M., Gilmore, J.H., Lin, W., Gerig, G., 2005. Automatic segmentation of MR images of the developing newborn brain. Medical Image Analysis 9, 457-466.
[43] Salembier, P., Oliveras, A., Garrido, L., 1998. Antiextensive connected operators for image and sequence processing. IEEE Transactions on Image Processing 7, 555-570.

[44] Samaille, T., Fillon, L., Cuingnet, R., Jouvent, E., Chabriat, H., Dormont, D., Colliot, O., Chupin, M., 2012. Contrast-based fully automatic segmentation of white matter hyperintensities: method and validation. PloS One 7, e48953.

[45] Serra, J., 1982. Image Analysis and Mathematical Morphology. volume 1. Academic Press, New York.

[46] Setänen, S., Lehtonen, L., Parkkola, R., Aho, K., Haataja, L., 2016. Prediction of neuromotor outcome in infants born preterm at 11 years of age using volumetric neonatal magnetic resonance imaging and neurological examinations. Developmental Medicine \& Child Neurology 28, 721-727.

[47] Shi, F., Fan, Y., Tang, S., Gilmore, J.H., Lin, W., Shen, D., 2010. Neonatal brain image segmentation in longitudinal MRI studies. NeuroImage 49, 391400 .

[48] Tarjan, R., 1975. Efficiency of a good but not linear set union algorithm. Journal of the ACM 22, 215225 .

[49] Tilea, B., Alberti, C., Adamsbaum, C., Armoogum, P., Oury, J., Cabrol, D., Sebag, G., Kalifa, G., Garel, C., 2009. Cerebral biometry in fetal magnetic resonance imaging: new reference data. Ultrasound in Obstetrics \& Gynecology 33, 173-181.

[50] Victora, C.G., Requejo, J.H., Barros, A.J., Berman, P., Bhutta, Z., Boerma, T., Chopra, M., de Francisco, A., Daelmans, B., Hazel, E., et al., 2016. Countdown to 2015: a decade of tracking progress for maternal, newborn, and child survival. The Lancet 387 , 2049-2059.

[51] Vincent, L., 1993. Grayscale area openings and closings, their efficient implementation and applications, in: First Workshop on Mathematical Morphology and its Applications to Signal Processing, pp. 22 27. 
[52] Vincent, L., 1994. Morphological area openings and closings for grey-scale images, in: Shape in Picture. Springer, pp. 197-208.

[53] Viola, A., Confort-Gouny, S., Schneider, J., Le Fur, Y., Viout, P., Chapon, F., Pineau, S., Cozzone, P., Girard, N., 2011. Is brain maturation comparable in fetuses and premature neonates at term equivalent age? American Journal of Neuroradiology 32, 1451-1458.

[54] Wang, L., Gao, Y., Shi, F., Li, G., Gilmore, J.H., Lin, W., Shen, D., 2015. LINKS: Learning-based multisource IntegratioN frameworK for Segmentation of infant brain images. NeuroImage 108, 160-172.

[55] Wang, S., Kuklisova-Murgasova, M., Hajnal, J.V., Ledig, C., Schnabel, J.A., 2016. Regression analysis for assessment of myelination status in preterm brains with magnetic resonance imaging, in: IEEE 13th International Symposium on Biomedical Imaging (ISBI), pp. 278-281.

[56] Wang, S., Murgasova, M., Schnabel, J.A., 2012. An atlas-based method for neonatal MR brain tissue segmentation. MICCAI Grand Challenge: Neonatal Brain Segmentation (NeoBrainS12), 28-35.

[57] Warfield, S.K., Kaus, M., Jolesz, F.A., Kikinis, R., 2000. Adaptive, template moderated, spatially varying statistical classification. Medical Image Analysis $4,43-55$.

[58] Weisenfeld, N.I., Warfield, S.K., 2009. Automatic segmentation of newborn brain MRI. NeuroImage 47, 564-572.

[59] Wen, W., Sachdev, P., 2004. The topography of white matter hyperintensities on brain MRI in healthy 60-to 64-year-old individuals. NeuroImage $22,144-154$.

[60] Woodward, L.J., Anderson, P.J., Austin, N.C., Howard, K., Inder, T.E., 2006. Neonatal MRI to predict neurodevelopmental outcomes in preterm infants. New England Journal of Medicine 355, 685694.
[61] Wu, J., Avants, B., 2012. Automatic registrationbased segmentation for neonatal brains using ANTs and atropos. MICCAI Grand Challenge: Neonatal Brain Segmentation (NeoBrainS12), 36-43.

[62] Xu, Y., Carlinet, E., Géraud, T., Najman, L., 2015. Efficient computation of attributes and saliency maps on tree-based image representations, in: International Symposium on Mathematical Morphology, Springer. pp. 693-704.

[63] Xu, Y., Géraud, T., Najman, L., 2012. Context-based energy estimator: Application to object segmentation on the tree of shapes, in: IEEE International Conference on Image Processing, pp. 1577-1580.

[64] Xue, H., Srinivasan, L., Jiang, S., Rutherford, M., Edwards, A.D., Rueckert, D., Hajnal, J.V., 2007. Automatic segmentation and reconstruction of the cortex from neonatal MRI. NeuroImage 38, 461477. 